\title{
Multi-wavelength mock observations of the WHIM in a simulated galaxy cluster
}

\author{
Susana Planelles ${ }^{1 \star}$, Petar Mimica ${ }^{1}$, Vicent Quilis ${ }^{1,2}$, Carlos Cuesta-Martínez ${ }^{1}$ \\ ${ }^{1}$ Departament d'Astronomia i Astrofísica, Universitat de València, c/ Dr. Moliner, 50, 46100 - Burjassot (Valencia), Spain \\ 2 Observatori Astronòmic, Universitat de València, E-46980 Paterna (Valencia), Spain
}

7 August 2021

\begin{abstract}
About half of the expected total baryon budget in the local Universe is 'missing'. Hydrodynamical simulations suggest that most of the missing baryons are located in a mildly overdense, warm-hot intergalactic medium (WHIM), which is difficult to be detected at most wavelengths. In this paper we explore multi-wavelength synthetic observations of a massive galaxy cluster developed in a full Eulerian-AMR cosmological simulation. A novel numerical procedure is applied on the outputs of the simulation, which are post-processed with a full-radiative transfer code that allows to compute the change of the intensity at any frequency along the null-geodesic of photons. We compare the emission from the whole inter-galactic medium (IGM) and from the WHIM component (defined as the gas with a temperature in the range $10^{5}-10^{7} \mathrm{~K}$ ) at three observational bands associated to thermal X-rays, thermal and kinematic Sunyaev-Zel'dovich effect, and radio emission. The synthetic maps produced by this procedure could be directly compared with existing observational maps and could be used as a guide for future observations with forthcoming instruments. The analysis of the different emissions associated to a high-resolution galaxy cluster is in broad agreement with previous simulated and observational estimates of both gas components.
\end{abstract}

Key words: cosmology: methods: numerical - galaxies: cluster: general - X-ray: galaxies.

\section{INTRODUCTION}

Observations of the cosmic microwave background (CMB; e.g. Planck Collaboration et al. 2016), primordial nucleosynthesis calculations (e.g. Kirkman et al. 2003) and Ly $\alpha$ absorption systems (Rauch 1998) have reported that about a 5 per cent of the total energy content of the Universe is in the form of baryons. At high redshift, $z \gtrsim 2$, the amount of gas measured from Ly $\alpha$ observations and contained in galaxies accounts for the full cosmic baryon budget. However, in the local Universe, while the Ly $\alpha$ forest can account for about one third of the low-z baryons, most part of them remain "missing" (Fukugita et al. 1998; Cen and Ostriker 1999b; Bregman 2007; Bertone et al. 2008).

Cosmological simulations predict that, according to the hierarchical model of cosmic structure formation, tiny density fluctuations in the primordial density field evolved into the large scale structure we observe today: an intricate network of voids, sheets and filaments connecting the largest clusters of galaxies (e.g. Kravtsov and Borgani 2012; Planelles et al. 2015, for reviews). As the cosmic evolution proceeds, gravitational shock waves propagate from collapsing and central regions to the surrounding en-

* e-mail: susana.planelles@uv.es vironment, heating in a efficient way the intra-galactic medium (IGM). While in the largest clusters of galaxies the intra-cluster medium (ICM) can be gravitationally heated up to temperatures of $10^{7}-10^{8} \mathrm{~K}$, mildly overdense regions, such as filaments, can only reach temperatures of $10^{5}-10^{7} \mathrm{~K}$ (e.g. Cen and Ostriker 1999b; Davé et al. 2001; Shull et al. 2012). This warm-hot intergalactic medium (WHIM) is thought to contain about 50 per cent of the total low-z cosmic baryons. The WHIM is expected to be located mainly in filaments but also around rich clusters and between pairs of merging clusters. However, given the typically moderate WHIM densities (from $\sim 4 \times 10^{-6}$ to $\sim 10^{-4} \mathrm{~cm}^{-3}$ ) and temperatures, its emission in the UV and soft X-ray bands, due to thermal Bremsstrahlung, is difficult to be detected by current observational facilities (e.g. Paerels et al. 2008). Moreover, at these temperatures, the IGM is fully ionized and contributes significantly to the X-ray background (XRB) in the form of a soft diffuse emission. In a similar way, absorption features from the WHIM are also too weak to be easily resolved (e.g. Richter et al. 2008).

Despite the low surface brightness associated to the WHIM, there have been claims of detection in X-ray analyses, either in absorption or in emission, such as those along the line of sight of distant quasi-stellar radio sources (e.g. Fang et al. 2007; Zappacosta et al. 2010; Nicastro et al. 2013) or in the gaseous filaments 
connecting merging clusters (e.g. Scharf et al. 2000; Finoguenov et al. 2003; Werner et al. 2008; Nicastro et al. 2010; Eckert et al. 2015). However, since $X$-ray surface brightness is proportional to the square of the gas density, X-ray observations are more suited to explore high-density regions, usually associated to the hot ICM within the cluster virial radius.

Besides X-ray observations, galaxy clusters can also be observed at millimeter wavebands through the Sunyaev-Zel'dovich effect (SZ; Sunyaev and Zel'dovich 1972, 1980). There exist a thermal (tSZ) and a kinematic (kSZ) SZ effect. The tSZ effect is the gain in energy of CMB photons when they are scattered, along the line of sight, by high-energy ICM electrons. Given that this effect is proportional to the gas density, these observations are crucial to reveal the physics of cluster outskirts (see Reiprich et al. 2013, for a review). In this regard, the diffuse WHIM gas component has been claimed to be detected through the tSZ effect in the microwave band (Planck Collaboration et al. 2013). On the other hand, when galaxy clusters present bulk motions, a second-order signal generated by the interaction between CMB photons and ICM electrons is the kSZ effect. In this case, the CMB photons can gain or lose energy depending on whether the ICM gas is approaching or going away from the observer. Recently, this kinematic Sunyaev-Zel'dovich effect has been also employed to claim the detection of all the missing baryons within and around the central galaxies in the Sloan Digital Sky Survey (Hernández-Monteagudo et al. 2015).

Although most of the emission from clusters is associated to thermal processes, non-thermal cluster radiation plays also a significant role (e.g. Brunetti and Jones 2014, for a recent review). Indeed, observations of radio haloes and radio relics (e.g. Ferrari et al. 2008, for a review) are associated to extended synchrotron radio emission from clusters. This radio emission originates as a consequence of structure formation shocks accelerating relativistic particles which, in the presence of a magnetic field, can emit synchrotron radiation. Therefore, non-thermal phenomena provide information on the pressure of the thermal gas, the distribution of shock waves and the level of ICM magnetic fields $(\sim 0.1-1 \mu \mathrm{G}$; Feretti and Giovannini 2008). Hence, the detection of accretion shocks around cosmic filaments would be essential to deepen our understanding of the WHIM component. Moreover, in addition to radio emission, it has been also claimed the existence of a hard $\mathrm{X}$-ray cluster emission due to inverse Compton scattering of relativistic ICM electrons and CMB photons (e.g. Rephaeli et al. 2008 , for a review). However, the origin and detection of this nonthermal emission are still controversial (e.g. Fusco-Femiano et al. 2004; Sanders et al. 2004, 2005; Eckert et al. 2008; Molendi and Gastaldello 2009; Pérez-Torres et al. 2009).

From a theoretical point of view, the first attempts to "observe" the WHIM component came from hydronamical simulations (e.g. Cen and Ostriker 1999b; Davé et al. 2001), which predicted that the WHIM can contribute up to 40 per cent of the total XRB emission (e.g. Croft et al. 2001; Roncarelli et al. 2006), with most of it coming from $z<0.9$ (see also Kravtsov et al. 2002; Ursino and Galeazzi 2006). Further numerical studies have put a significant effort in analysing the properties of the WHIM component depending on the inclusion and treatment of different physical processes such as radiative cooling, star formation and its associated feedback, chemical enrichment, or magnetic fields (e.g. Cen and Ostriker 2006; Cen and Fang 2006; Bertone et al. 2010b,a; Tornatore et al. 2010; Roncarelli et al. 2012; Vazza et al. 2015b; Gheller et al. 2015). Several numerical studies have also exploited the complementarity of X-ray and SZ properties of the WHIM (e.g. Hernández-Monteagudo et al. 2006; Roncarelli et al.
2007; Ursino et al. 2014), confirming that such a combination represents a promising tool to study future WHIM observations. Simulations have been also employed to explore the non-thermal emission from galaxy clusters (e.g. Pfrommer et al. 2008; Hoeft et al. 2008; Battaglia et al. 2009; Brown 2011; Araya-Melo et al. 2012; Vazza et al. 2015b,a, 2016; Basu et al. 2016).

In the near future, a new generation of telescopes will significantly widen our understanding of the main properties and distribution of the WHIM. In fact, the highly-improved capabilities of these upcoming facilities will certainly unveil a whole new picture of the WHIM in several different wavelength bands like the ones associated with the emissions in X-rays, SZ and radio. In this context, our aim is to use a high-resolution simulated galaxy cluster to produce synthetic detailed maps - in the aforementioned bands directly comparable with existing or future planned and, therefore, improve our knowledge on the WHIM component by getting closer the theoretical and the observational planes. To do so, we identify the WHIM component (defined as the gas with a temperature in the range $10^{5}-10^{7} \mathrm{~K}$ ) at several locations from the cluster centre to its outskirts. Once this is done, we use a novel approach to post-process the data from the simulation by means of a relativistic full-radiative transfer code. This code computes the change in the intensity along each line of sight by integration of the null-geodesic of photons. The code is capable of computing the self-absorption, and we use that capability to verify that the absorption is negligible, so that we are justified in running the code in post-processing and in neglecting any radiation back-reaction on the gas. In this paper, we focus on the analysis of the thermal X-ray emission of the cluster (in the soft and hard X-ray bands), the associated tSZ and kSZ effects, and the corresponding radio emission. We wish to emphasize that all bands are treated consistently by the same code, thus avoiding the usage of different numerical approaches for each separate wavelength. The results derived from this approach are compared with previous existing data, both numerical and observational.

This paper is organised as follows. In Section 2 we briefly introduce the numerical codes employed in our study, that is, an Eulerian cosmological code to perform the hydrodynamical simulation and a radiative transfer code to process the outputs of the simulation and compute the associated emission in different bands. Moreover, a description of the main properties of our main simulated cluster is also included. In Section 3 we present our results on the different X-rays, SZ and radio emissions of the WHIM and the IGM. Finally, in Section 4, we summarise and discuss our main findings. Besides, while Appendix A is devoted to discuss how the contribution from metal lines affects the estimation of the X-ray emission, Appendix B shows instead how relevant is to consider the relativistic corrections when computing the SZ signal.

\section{NUMERICAL DETAILS}

In this section we present the main numerical details of the codes we have employed in the present analysis, namely, the cosmological code MASCLET (Quilis 2004) and the radiative transfer code SPEV (Mimica et al. 2009; Cuesta-Martínez et al. 2015; Mimica et al. 2016). Here, we only provide a brief description of the main features relevant for the present study, while we refer the reader to the corresponding references for further details. 


\subsection{The cosmological simulation}

In this paper we analyse the outcomes of a hydrodynamical simulation performed with the cosmological code MASCLET (Quilis 2004). MASCLET is an Eulerian code, with an adaptive mesh refinement (AMR) scheme, that couples high-resolution shock capturing techniques to resolve the evolution of the gaseous component of the Universe with an N-body scheme to evolve the dark matter component.

Our simulation accounts for a flat $\Lambda C D M$ universe with cosmological parameters: $\Omega_{\Lambda}=\Lambda / 3 H_{o}^{2}=0.69, \Omega_{m}=0.31$, $\Omega_{b}=0.048, h=H_{0} / 100 \mathrm{~km} \mathrm{~s}^{-1} \mathrm{Mpc}^{-1}=0.678, \sigma_{8}=0.82$ and $n_{s}=0.96$.

The simulation domain, a cubical box with a comoving side length of $40 \mathrm{Mpc}$, has been discretised using $128^{3}$ cubical cells in the coarse (lowest resolution) level. In this simulation we allow for a maximum of 9 refinement levels (see Navarro-González et al. 2013; Quilis et al. 2017, for further details on the refinement procedure), providing a peak physical spatial resolution of $\sim 610 \mathrm{pc}$ at $z=0$. As for the dark matter particles, the best mass resolution is $\sim 2 \times 10^{6} M_{\odot}$, which is equivalent to distributing $1024^{3}$ particles throughout the computational volume.

Using a CDM transfer function (Eisenstein and $\mathrm{Hu}$ 1998), the initial conditions of our simulation were set up at $z=100$. Moreover, following the ideas presented in Hoffman and Ribak (1991), we have performed a constrained realization in order to reproduce a big galaxy cluster at the centre of our simulated volume.

Besides gravity and hydrodynamics, the simulation includes additional physical processes such as inverse Compton and freefree cooling, atomic and molecular cooling for a primordial gas, and UV heating (Haardt and Madau 1996). With the purpose of computing the abundances of each specie, the gas is considered to be optically thin and in ionization equilibrium, but not in thermal equilibrium (Katz et al. 1996; Theuns et al. 1998). We employ metallicity dependent tabulated cooling rates from Sutherland and Dopita (1993) and we truncate the cooling curve below a temperature of $10^{4} \mathrm{~K}$. In addition, our simulation includes star formation, according to the models by Yepes et al. (1997) and Springel and Hernquist (2003), and feedback from supernova (SN) type II. However, feedback from stellar winds, SN type Ia, or active galactic nuclei (AGN) are not accounted for.

\subsection{Computing the emission}

Obtaining proper observables from hydrodynamical simulations is not a trivial task. In order to reproduce the pictures obtained by an observer at $z=0$, we need to consider all the matter contributing to the integrated signal up to a given cosmic time. There have been several studies trying to overcome this difficulty, for instance, by reconstructing the past light cone as observed at $z=0$ (e.g. Roncarelli et al. 2012). Despite the improvement of this approach, it is still an approximated way to deal with this issue. In our study, we use instead a full-radiative transfer code to compute the thermal and non-thermal emission in different X-rays, SZ, and radio bands. We note that our method uses a code developed for the radiative transfer in relativity (see below), so that it follows the emission along the null-geodesics and can provide consistent multiwavelength and multi-epoch synthetic observations. In this case we use the same null-geodesics to also compute the SZ effect, so that we consistently compute all the observational signals. Moreover, as we explain below, the method is well-tested and converges with the increasing spatial and temporal simulation resolution.
To compute the emission from MASCLET snapshots we use in post-processing the code SPEV (Mimica et al. 2009). Although it was originally designed to process the outputs of a relativistic hydrodynamics simulation and compute the non-thermal emission, it has since been expanded to include the thermal emission (Cuesta-Martínez et al. 2015). In this work we compute both thermal and non-thermal emission using a two-step procedure. First, the raw output from MASCLET is preprocessed to select the numerical cells that satisfy the criteria for the emission. Then, the preprocessed data is fed to the post-processor that generates a multi-frequency image. SPEV first processes each cell to determine which pixels of the image can observe it. It then computes the emission from each zone at the proposed frequencies, and stores it in memory reserved for the lines of sight leading to those pixels, together with the information about the zone position, geometry, and velocity, as well as the electron density and temperature (for the purpose of computing the SZ effect, see Sec. 2.3). We assume that the pixel at the origin of the image (right in its geometrical centre) observes the centre of the simulation box when it is at $z=0.36$ (see Section 2.4 for further details on this choice). This determines the range of redshifts and positions of the cells that can be observed simultaneously with the origin. In order to obtain the continuous spacetime coverage, we interpolate the positions of the cell corners between snapshots assuming a linear motion and taking into account the Hubble flow and their peculiar velocity. This interpolation in spacetime allows SPEV to deal with an arbitrary numerical resolution, as well as with the arbitrary frequency with which simulation snapshots are stored. For the numerical tests that demonstrate the convergence of SPEV with increasing temporal and spatial numerical resolution see Mimica et al. (2016).

After all the zones have been post-processed, SPEV solves the radiative transfer equation for each pixel in order to compute the observed intensity at a frequency $\nu$ :

$$
\mathrm{d} I_{\nu} / \mathrm{d} s=j_{\nu}-\alpha_{\nu} I_{\nu},
$$

where $I_{\nu}, j_{\nu}$ and $\alpha_{\nu}$ are the intensity, emissivity and absorption coefficients, respectively, and $s$ is the path along the line of sight. In practice, the line of sight is composed of finite segments, each corresponding to an intersection with a numerical cell. We assume that $j_{\nu}$ and $\alpha_{\nu}$ are constant in each segment and can easily compute the final intensity by sorting the segments in the order of decreasing distance from the observer and then analytically solving Eq. 1 in each segment, taking as the initial value for $I_{\nu}$ the outgoing intensity from the previous segment. The outgoing intensity from the last segment is then the observed intensity of the pixel. The incoming intensity into the first (furthest away from the observer) segment is assumed to be zero (except when computing SZ, where we assume the CMB intensity, see Sec. 2.3). We note that the algorithm described here is different from the light-cones scheme used in some previous works (e.g., Roncarelli et al. 2006) since it does not use stacking at different redshifts, but rather produces a consistent image in the observer frame by creating a continuous spacetime representation of the simulation regardless of its actual spatial and temporal resolution. For more details about the SPEV imaging algorithm see Mimica et al. (2016).

\subsubsection{Thermal emission}

The free-free thermal Bremsstrahlung is the dominant emission process at the characteristic ICM temperatures. However, as we go to lower temperatures the emission through metal lines also becomes relevant (e.g. Böhringer and Werner 2010). Actually, most 
of the emission associated to the WHIM in the soft X-ray band is due to line emission (e.g. Bertone et al. 2008, 2010b). Therefore, a proper implementation of metal enrichment is crucial to perform an accurate determination of the observational IGM and WHIM properties.

In the current analysis, following the results presented in Croft et al. (2001), and in a similar way to previous studies (e.g. Branchini et al. 2009; Takei et al. 2011), we estimate the gas metallicity in solar units according to $Z=\min \left(0.3,0.005\left(1+\delta_{g}\right)^{1 / 2}\right)$, where $\delta_{g}=\rho_{\text {gas }} /\left\langle\rho_{\text {gas }}\right\rangle-1$ and $\rho_{\text {gas }}$ is the gas density. This metallicity estimate, computed in post-processing, is provided to SPEV in order to compute the thermal X-ray emission as contributed by both the free-free Bremsstrahlung and the line emission. As explained in Takei et al. (2011), although this metallicity estimate is not totally self-consistent with the thermodynamical state of the gas, it avoids the underestimation of the metal abundance in low-density regions which, due to the characteristics of our simulation, are worse resolved.

In the default version of SPEV, in order to compute the thermal X-ray emission, we assumed that the dominant process is the free-free thermal Bremsstrahlung and use the Kirchhoff's law to compute the absorption coefficient (see Appendix A1 of CuestaMartínez et al. 2015, for a detailed explanation on how the thermal emission is implemented in SPEV. Furthermore, we note that the Gaunt factor tables have been updated with the ones computed by van Hoof et al. $(2014,2015))^{1}$. However, this is not an accurate assumption to compute the thermal X-ray emission from galaxy clusters in the energy regime where line emission from metals have a significant contribution. Therefore, in this work, to compute the $\mathrm{X}$-ray images having also into account the contribution from lines, we need to be able to model the plasma emission in a large dynamic range of parameters. In order to do so, we use the publicly available code CLOUDY (version 17.00; Ferland et al. 2017) to construct a large interpolation table of the X-ray emission in different energy bands. In Appendix A we provide a detailed explanation about the computation procedure, as well as a comparison between this and the default SPEV thermal emission method. According to our tests, the method based on CLOUDY tables allows us to reliably predict the X-ray emission from low-density and low-temperature media.

In practice, for each model generated with CLOUDY (see Appendix A for details) we assume thermal equilibrium, use the $C M B$ and HM0 5 CLOUDY commands to generate the CMB and UV background radiation fields (Haardt and Madau 1996), and save the diffuse continuum. We then integrate the continuum in the soft $(0.5-2$ $\mathrm{keV})$ and hard $(2-10 \mathrm{keV}) \mathrm{X}$-ray energy bands. In Section 3.2 we will analyse the results obtained using this method when we compute synthetic X-ray maps at the soft and at the hard X-ray energy bands for the IGM and the WHIM.

\subsubsection{Non-thermal emission}

The nature, strength and distribution of cosmic magnetic fields is still highly uncertain (e.g. see Dolag et al. 2008, for a review). It is beyond the scope of the present work to perform a detailed analysis of different magnetisation models in order to constrain current uncertainties (e.g. see Vazza et al. 2017, 2018, for recent works on different ICM magnetic field models). We pretend instead to show

${ }^{1}$ By comparing the emission maps with and without absorption we verified that it is negligible in the scenario we studied. the capabilities of SPEV in generating radio images from cosmological simulations. Therefore, in order to estimate the non-thermal emission, we need to adopt some simplifications. In particular, following the model employed in some recent works (e.g. Hoeft et al. 2008; Araya-Melo et al. 2012), we assume that the magnetic field, $B$, satisfies flux conservation and is given by

$$
\frac{B}{B_{\text {ref }}}=\left(\frac{n}{10^{-4} \mathrm{~cm}^{-3}}\right)^{2 / 3}
$$

where $B_{\text {ref }}=0.1 \mu \mathrm{G}$ and $n$ stands for the gas number density in the post-shock region. We only take into account the non-thermal emission when $B<1 \mu \mathrm{G}$ (e.g. Feretti and Giovannini 2008) ${ }^{2}$. Moreover, this emission is computed only from those zones where the Mach number, $\mathcal{M}$, is greater than 1.01 (see Planelles and Quilis 2013; Martin-Alvarez et al. 2017, for details on the shock-finding algorithm employed to compute the strength of shocks).

In this work we do not implement any transport scheme for the non-thermal electrons. We use the formalism of Miniati (2001) and Enßlin et al. (2007) to compute the power-law index $\left(\alpha_{i n j}\right)$ and the lower energy cutoff $\left(\gamma_{\text {min }}\right)$ for the non-thermal electron distribution:

$$
\begin{gathered}
\alpha_{i n j}=2\left(\mathcal{M}^{2}+1\right) /\left(\mathcal{M}^{2}-1\right) \\
\gamma_{m i n}=x_{i n j} \sqrt{2 k T /\left(m_{p} c^{2}\right)},
\end{gathered}
$$

where $T$ is the temperature of the shocked gas and $m_{p}$ and $c$ are the proton mass and the speed of light. We set $x_{i n j}$ to 3.5 (Enßlin et al. 2007). The upper cut-off is determined by balancing the synchrotron cooling and the acceleration timescales:

$$
\gamma_{\max }=\left(\frac{3 m_{e}^{2} c^{4}}{4 \pi e^{3} B}\right)^{1 / 2}
$$

where $m_{e}$ and $e$ are the electron mass and charge, respectively. The synchrotron emission is computed using the standard formulae (e.g., Rybicki and Lightman 1979) and employing an efficient numerical method (as explained in Sec. 4 of Mimica et al. 2009).

In Section 3.4 we will discuss and compare the results obtained with our reference model (also labelled as 'model B1'), given by Eq. 2, with those derived when we assume the magnetic field to be in a fraction of equipartition with the gas internal energy density, i.e.

$$
B^{2} / 8 \pi=\epsilon_{B} P,
$$

where $P$ is the thermal pressure and $\epsilon_{B}$ is a proportionality constant (we assume $\epsilon_{B}=10^{-3}$ ). We will labelled this model as 'model B2' and, as with our reference model, we will only take into account the non-thermal emission when $B<1 \mu \mathrm{G}$.

\subsection{Computing the $\mathrm{SZ}$ effect}

In an intermediate step before producing the final image, SPEV needs to organize the data along the lines of sight, so that each line of sight contains zones that are simultaneously observed in the center of a particular pixel. We use these line of sight data structures to also compute the thermal and kinematic SZ effects in the nonrelativistic approximation (see Birkinshaw 1999; Carlstrom et al. 2002; Colafrancesco 2007, for reviews).

As mentioned in the previous section, for each pixel we

2 We performed tests with different values of the upper limit (up to $5 \mu \mathrm{G}$ ), but found no significant changes in the final convolved images shown in Section 3.4 
store the electron density, the electron temperature, and the fluid velocity in all the contributing numerical cells. To compute the SZ effect we have modified the final phase of the SPEV algorithm, when the pixel intensities are computed, as follows. First, we initialize the pixel intensities to the CMB intensity, that is, $I_{\nu}=2 h \nu^{3} / c^{2} /\left(e^{x}-1\right)$, where $h$ is the Planck constant, $x:=$ $h \nu / k T_{C M B}$ is a dimensionless frequency, $k$ is the Boltzmann constant, and $T_{C M B}=2.72548 \mathrm{~K}$ (e.g. Fixsen 2009). Therefore, the change in the intensity due to the thermal SZ effect is given by

$$
\Delta I_{\nu, t h}=\frac{x e^{x}}{e^{x}-1}\left[x \frac{e^{x}+1}{e^{x}-1}-4\right] I_{\nu} \int \mathrm{d} s \frac{k T_{e}}{m_{e} c^{2}} n_{e} \sigma_{T}
$$

where $T_{e}$ and $n_{e}$ are the electron temperature and number density, and $\sigma_{T}$ is the Thomson cross-section. The integral in Eq. 7 is computed using the same numerical method that is used for solving Eq. 1.

On the other hand, the kinematic SZ effect, contributed by the ICM bulk motion, is computed according to the following equation:

$$
\Delta I_{\nu, k}=\frac{x e^{x}}{e^{x}-1} I_{\nu} \int \mathrm{d} s n_{e} \sigma_{T} \frac{v_{\|}}{c},
$$

where $v_{\|}$, the component of the gas velocity parallel to the line of sight, can be either positive or negative depending on whether the gas element is moving away or approaching to the observer.

In the following, we use Eqs. 7 and 8 to compute separately the thermal and kinematic SZ contributions, as well as the total SZ effect (see Sec. 3.3):

$$
\Delta I_{\nu} / I_{\nu}=\Delta I_{\nu, t h} / I_{\nu}+\Delta I_{\nu, k} / I_{\nu}
$$

For a value of $T_{C M B}=2.72548 \mathrm{~K}$, the tSZ signal given by Eq. 7 shows its minimum and maximum intensities at frequencies $\nu \sim 128 \mathrm{GHz}$ and $369 \mathrm{GHz}$, respectively. On the contrary, at $\nu \sim 217 \mathrm{GHz}$ the tSZ effect vanishes and the total SZ signal is dominated by the kinematic contribution. Therefore, the analysis of the SZ effect at these three characteristic frequencies is very useful to disentangle the thermal and kinematic contributions to the total SZ signal and to derive the cluster thermal distribution (e.g. Kay et al. 2008; Prokhorov et al. 2010, 2011).

We note that equations presented above are valid to estimate the SZ effect in the non-relativistic approximation and, therefore, in this work we will compute the different SZ signals without taking into account the relativistic correction. In a future work, we plan to include these corrections in SPEV and to perform a detailed analysis on the accuracy of the results for a larger sample of galaxy clusters. For the moment, in order to have an idea of the significance of the approximation and the performance of SPEV, we present in Appendix B a comparison between the results obtained with SPEV for the total SZ signal and those obtained with the SZPACK ${ }^{3}$ code (Chluba et al. 2012, 2013) at different frequencies. SZPACK is a publicly available library that, taking into account relativistic corrections, provides a precise estimation of the SZ effects in massive galaxy clusters for a wide range of electron temperatures and cluster peculiar velocities. A deeper analysis of the effects of the relativistic corrections on the SZ effect of galaxy clusters is out of the scope of the present paper and, therefore, we defer the reader to more suitable works on this purpose (e.g. Itoh et al. 1998; Nozawa et al. 2005).

\footnotetext{
${ }^{3}$ http://www.Chluba.de/SZpack
}

\subsection{The simulated cluster}

In order to identify the sample of dark matter haloes in our simulation, we apply the spherical overdensity halo finder ASOHF (see Planelles and Quilis 2010; Knebe et al. 2011, for further details). Within our simulated volume, we find a sample of $\sim 44$ haloes with virial masses ${ }^{4}$ larger than $10^{13} M_{\odot}$. However, for the purpose of the present study, we are only interested in the analysis of the main central halo developed in our simulation. The excellent numerical resolution in this central region allows us to describe in detail the distribution of matter and its associated emission from the centre of the main halo out to its outskirts. At $z=0$, the central halo developed in the simulated volume, which is also the largest one, has a virial mass of $M_{v i r} \sim 4.3 \times 10^{14} M_{\odot}$ and a virial radius of $R_{v i r} \sim 1.98 \mathrm{Mpc}$. However, motivated by recent observations of massive galaxy clusters (see e.g. Eckert et al. 2015), in the present study we will take as a target the main progenitor of this simulated cluster but at $z=0.33$, when it has $M_{v i r} \sim 3.2 \times 10^{14} M_{\odot}$ and $R_{\text {vir }} \sim 1.7 \mathrm{Mpc}$ and it is still the central and largest halo in the simulation.

Our cosmological simulation, which follows the cosmic evolution from $z=100$ down to $z=0$, generates a total of 80 snapshots non-uniformly distributed in redshift. These outputs from the cosmological code become the inputs for SPEV in order to compute the emission associated to the different gaseous components. However, since we are mainly interested in the WHIM emission and the contribution from high-z gas is expected to be negligible, we will only take into account the redshift interval $0.65<$ $z<0.33$, which corresponds to 11 discrete snapshots. We verified that no contribution to the emission comes from the snapshots with $z \geqslant 0.65$ or $z \leqslant 0.33$ and, therefore, to speed up the computation, we do not use the data from those snapshots as inputs for SPEV.

Therefore, unless otherwise stated, in the following we will analyse the $\mathrm{X}$-ray, $\mathrm{SZ}$ or radio signals coming from inside and from an extended region around our main central halo as observed at $z=0.33$. In order to do so, we will take into account all simulation snapshots that contain volumes that can be observed simultaneously with the central halo at $z=0.33$. Moreover, given the wide distribution of the WHIM and the typical extensions of recent WHIM studies (e.g. Eckert et al. 2015), we will consider a region with an amplitude of $5 \times R_{v i r}$ from the halo centre, which corresponds to an angular extension of around $0.6^{\circ}$ along each coordinate direction. Given the features of our simulation, this choice also ensures an excellent numerical resolution of the selected region throughout the redshift evolution.

Within our volume of interest we will consider the distribution and emission from two types of gas: the IGM, which includes all the gas cells within the selected volume, and the WHIM component, formed by those cells with a gas temperature in the range $10^{5} \mathrm{~K}<T<10^{7} \mathrm{~K}$. We note that the definition of the WHIM used in numerical studies is still somehow arbitrary: while some analyses define the WHIM according only to the gas temperature cut, as we do, (e.g. Roncarelli et al. 2006; Tornatore et al. 2010), some others include as well a condition on the gas density (e.g. Roncarelli et al. 2012; Ursino et al. 2014).

Given the lack of an efficient central source of feedback in our simulation, such as the one associated to a central AGN (see, e.g. Planelles et al. 2013, and references therein), our cluster is charac-

4 The viral mass, $M_{v i r}$, is the mass contained within a sphere of radius $R_{v i r}$ enclosing an average density equal to $\Delta_{v i r}$ (Bryan and Norman 1998) times the corresponding critical cosmic density. 

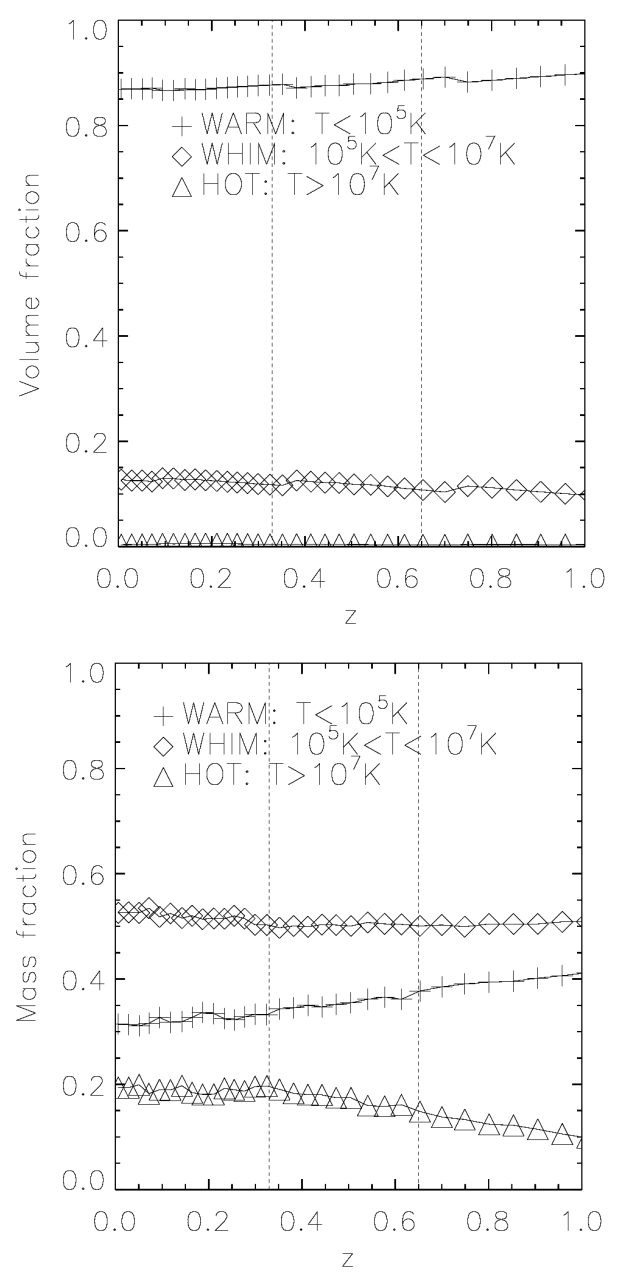

Figure 1. Redshift evolution of the fraction in volume (top panel) and in mass (bottom panel) occupied by different IGM gas components within the whole simulated volume. Vertical lines in both panels enclose the temporal region within which we will compute the cluster emission.

terized by an excessive X-ray emission in its central region. Therefore, in order to overcome this issue and avoid an spurious estimate of the corresponding emission, we have computed the cooling time associated to each gas element in our simulation and removed those gas cells with $t_{\text {cool }}<10^{10}$ years (Lufkin et al. 2000). This cut in cooling time slightly compensates for the strong central overcooling in the centre of our cluster.

We note that, when comparing with previous numerical analyses of the WHIM component, our study may suffer from different caveats such as the relatively small considered volume or the lack of an efficient AGN feedback source. However, we would like to point out that, on the one hand, the moderate volume is compensated by an excellent resolution throughout the analysed region and, in any case, it is in agreement with the real extension of some recent cluster observations (e.g. Eckert et al. 2015). On the other hand, although we do not include any AGN feedback model in our simulation, we compute the emission of each gas element along the line of sight with a full-radiative transfer code, obtaining a complete and fully consistent analysis of the WHIM at different wavebands.

\section{RESULTS}

\subsection{Distribution and evolution of different gas components}

Before analyzing in detail the characteristic WHIM emission through different physical processes, we study the global redshift and spatial distribution of different IGM gas components in our simulated volume.

Figure 1 shows the evolution since $z=1$ of the fractions in volume (top panel) and in mass (bottom panel) occupied by different IGM gas components within the whole $(40 \mathrm{Mpc})$ simulated box. In particular, we show separately the evolution of three different IGM regimes classified according to the gas temperature: the warm $\left(T<10^{5} K\right)$, the WHIM $\left(10^{5} K<T<10^{7} K\right)$, and the hot $\left(T>10^{7} K\right)$ gas components. Several interesting trends can be drawn from this figure. As expected, most of the volume is dominated by the warm $(\sim 90 \%)$ and the WHIM $(\sim 10 \%)$ gas phases, which are the most spread components throughout the redshift evolution. On the contrary, the hot gas component, mainly associated to collapsed regions such as galaxy clusters, represents only a few per cent of the simulated volume. In terms of mass we note that, as evolution proceeds and the largest cosmic structures begin to be formed, the amount of gas in the hot phase increases from $10 \%$ at $z=1$ up to $20 \%$ at $z=0$. Simultaneously, while the amount of gas in the warm component slightly decreases down to $30 \%$ at $z=0$, the amount of WHIM mildly augments from $50 \%$ at $z=1$ up to a value of $\sim 55 \%$ at the present epoch. These values are in broad agreement with the results obtained from previous numerical analyses (e.g. Cen and Ostriker 1999b). Nevertheless, we point out that the inclusion in simulations of different physical processes or the assumption of slightly different cosmologies may affect the obtained evolutions (e.g. Davé et al. 2001; Cen and Ostriker 2006; Tornatore et al. 2010; Ursino et al. 2014). On the other hand, although employing larger cosmological boxes allows for a larger number of group- and cluster-size haloes, the simulated box size does not significantly affect the baryon budget (e.g. Tornatore et al. 2010).

From now on, unless otherwise specified, we will focus on two types of gas: the whole IGM, formed by all the gas within our simulated volume, and the WHIM component, formed by the gas with $10^{5} \mathrm{~K}<T<10^{7} \mathrm{~K}$. We will analyse the emission from these two gas components in different wavebands and through different physical mechanisms. Moreover, as explained in Section 2.4, we will only consider a subvolume of radius $\sim 8.5$ comoving Mpc around the centre of the main halo. Within this volume, Fig. 2 shows the $3 \mathrm{D}$ distribution of the WHIM $\left(10^{5} K<T<10^{7} K\right)$ and the hot ICM $\left(T>10^{7} K\right)$ gas components. Both gas components have been selected according only to the gas temperature, whereas the color code stands for their corresponding densities in units of $\mathrm{M}_{\odot} / \mathrm{Mpc}^{3}$. From this figure, it is clear that the WHIM is distributed around the main cluster with a filamentary structure throughout the considered volume, whereas the ICM is confined to the more central cluster region.

For the sake of completeness, Fig. 3 shows the projection along the line of $\operatorname{sight}^{5}$ of the density maps associated to the IGM and the WHIM gas components within our volume of interest. In the IGM map (top panel), high-density regions (yellowish colors) are clearly correlated with the main haloes and subhaloes in our

5 Throughout the paper we will consider the line of sight as parallel to the $\mathrm{z}$-axis of the simulation box. We have verified that considering different projections does not have important implications on our conclusions. 


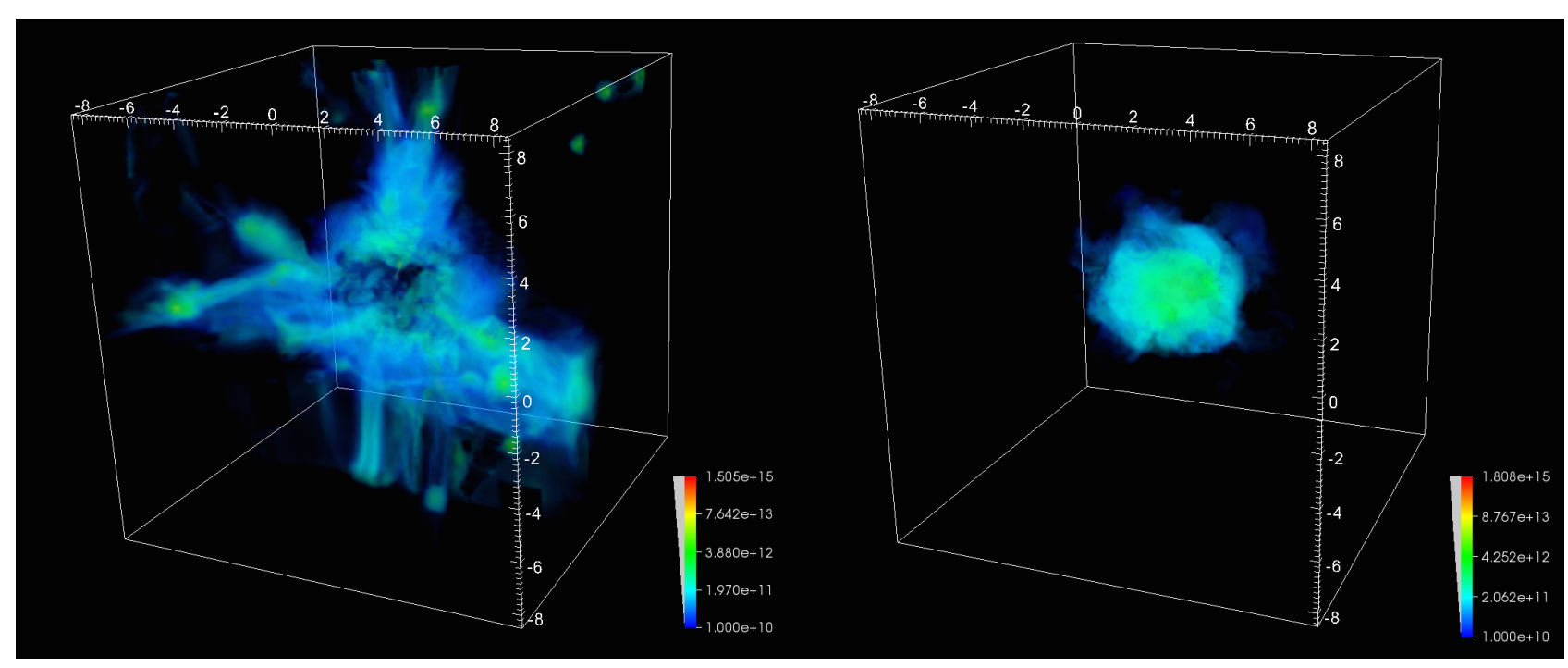

Figure 2. 3D distribution of the WHIM $\left(10^{5} K<T<10^{7} K\right.$; left panel) and ICM $\left(T>10^{7} K\right.$; right panel) gas components within a simulated volume of $\sim 17 \mathrm{Mpc}^{3}$ centered at the position of the most massive galaxy cluster at $z=0$. The color code stands for the gas density in units of $\mathrm{M}_{\odot} / \mathrm{Mpc}^{3}$.

simulation. As expected, due to the characteristics of our simulation and to the volume selection we have performed, the central region is dominated by the largest cluster. If we only take into account the WHIM component (bottom panel), the most massive (and hottest) virialized systems are almost completely removed and what remains is the distribution of the warm-hot gas, mainly associated to the smallest objects. This component is distributed throughout the whole volume with a tendency to reside around the location of the main halo, showing as well a more filamentary distribution that extends out to the limits of the image. However, we note that, due to projection effects, part of the filamentary structure is diluted with the diffuse background, making more difficult its detection (e.g. Croft et al. 2001; Roncarelli et al. 2006).

\subsection{Thermal X-ray emission}

As explained in Section 2.2, we employ SPEV in order to compute maps of thermal X-ray emission associated to the IGM and the WHIM components in the soft $(0.5-2 \mathrm{keV})$ and in the hard $(2.0-10.0 \mathrm{keV}) \mathrm{X}$-ray energy bands.

In the following, each map has been made in order to cover a region of $\sim 0.6$ squared degrees around the main central halo and is sampled with $3200^{2}$ square pixels. This provides a pixel size of $\sim 0.68^{\prime \prime}$, a value in line with the FWHM of $X M M$ Newton and Chandra point-spread functions (PSF). The forthcoming ATHENA $+{ }^{6}$ telescope, which has as one of its key science goals the detection and characterization of the WHIM (e.g. Nandra et al. 2013; Barcons et al. 2017), will have better capabilities and will provide a detection limiting flux of $10^{-17} \mathrm{erg} \mathrm{s}^{-1} \mathrm{~cm}^{-2}$ in the soft band. Therefore, as for the detection limit of our synthetic maps, we decide to adopt an optimistic intensity threshold of $10^{-17}$ $\mathrm{erg} \mathrm{s}^{-1} \mathrm{~cm}^{-2} \operatorname{arcmin}^{-2}$. However, we will not tune our maps with the response function or the particular capabilities of any specific observational facility.

According to this procedure, the synthetic X-ray emission maps, shown in the left and middle columns of Fig. 4, correspond

6 http://www.the-athena-X-ray-observatory.eu

\begin{tabular}{ccc}
\hline $\begin{array}{c}\text { Energy band } \\
(\mathrm{keV})\end{array}$ & $\begin{array}{c}\text { IGM } \\
\left(\mathrm{erg} \mathrm{s}^{-1} \mathrm{~cm}^{-2} \mathrm{deg}^{-2}\right)\end{array}$ & $\begin{array}{c}\text { WHIM } \\
\left(\mathrm{erg} \mathrm{s}^{-1} \mathrm{~cm}^{-2} \mathrm{deg}^{-2}\right)\end{array}$ \\
\hline $0.5-2$ & $\left(5.09_{+62.73}^{-0.80}\right) \times 10^{-13}$ & $\left(0.91_{+3.98}^{-0.44}\right) \times 10^{-13}$ \\
$2-10$ & $\left(4.73_{+68.98}^{-0.77}\right) \times 10^{-13}$ & $\left(0.71_{+1.29}^{-0.44}\right) \times 10^{-13}$ \\
\hline
\end{tabular}

Table 1. Median pixel values of the X-ray intensity maps shown in Fig. 4 for the IGM and the WHIM gas components at two different energy bands. Errors represent the 15 th and 85 th percentiles of each distribution. Median values and errors are obtained from all the pixels with intensity above $10^{-17} \mathrm{erg} \mathrm{s}^{-1} \mathrm{~cm}^{-2} \operatorname{arcmin}^{-2}$, which corresponds to $3.6 \times 10^{-14}$ $\operatorname{erg~s}^{-1} \mathrm{~cm}^{-2} \mathrm{deg}^{-2}$.

to the density distributions of the IGM and the WHIM displayed in Fig. 3. Qualitatively, these synthetic maps are in line with X-ray observations of the IGM. Indeed, as already shown in previous studies (e.g. Croft et al. 2001), we confirm that most of the IGM thermal emission is observed in the soft regime, being the hard band mainly contributed by the emission from the largest (hottest) groups and clusters. Moreover, the map obtained in the soft band highlights the existence of very small structures or density clumps with a considerable soft emission which are not visible in the hard regime. The emission from these structures is mainly contributed by metals, which tend to be located in mildly overdense warm/hot regions (e.g. Cen and Ostriker 1999a). As for the emission associated to the WHIM, there is a kind of geometrical characterization of the X-ray signals from IGM and WHIM at both energy bands: while the former is mainly connected to high-density regions and is dominant in the cluster core, the latter is mainly located in outer cluster regions around the cluster centre. Moreover, given its lower temperatures and densities, the hard emission associated to the WHIM seems fainter than the emission from the IGM (e.g. Croft et al. 2001; Roncarelli et al. 2006). In the soft band, however, the contribution from metals would highlight the more filamentary and extended emission from the WHIM.

Quantitatively, there is also a good match between the X-ray surface-brightness associated to our simulated cluster and recent observations of massive galaxy clusters (e.g. Eckert et al. 2015). 

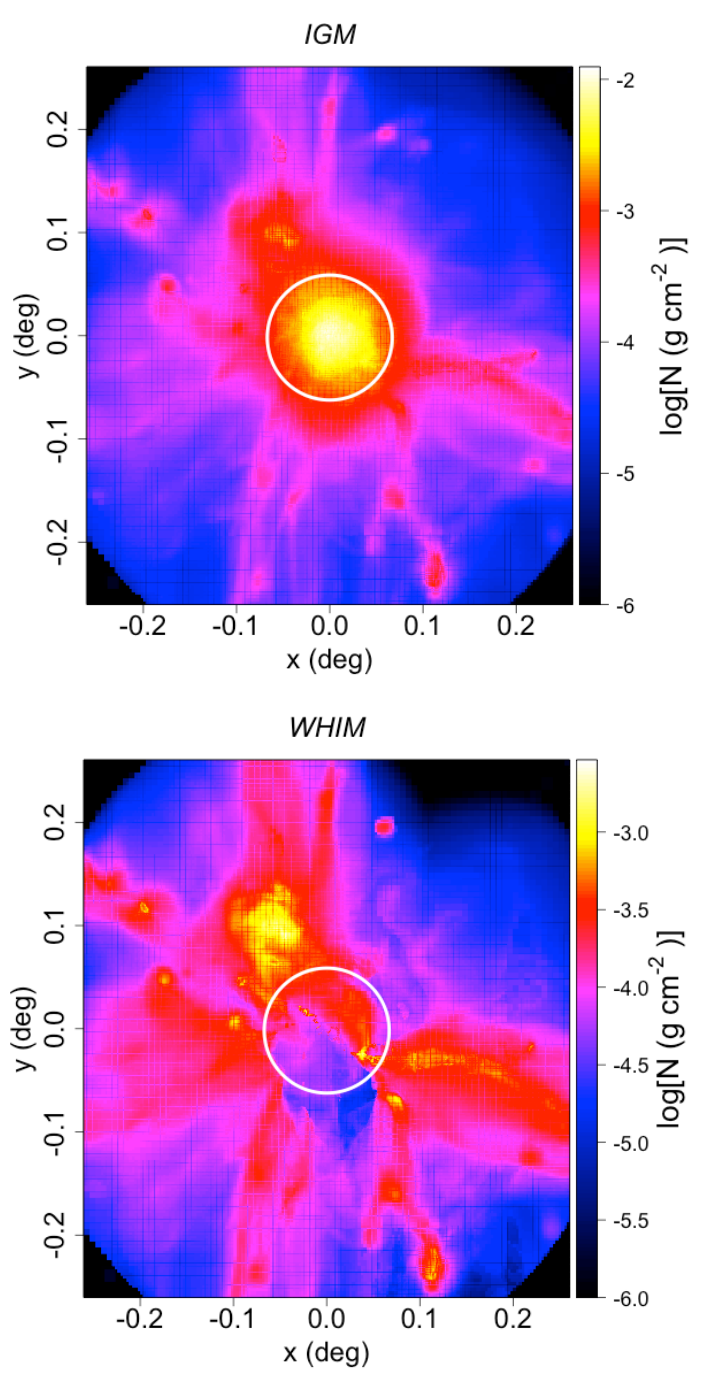

Figure 3. Surface density maps for the IGM and the WHIM gas components (top and bottom panels, respectively) within a region of $\sim 0.6^{\circ}$ around the main and central cluster in the simulation. The white circle represents the virial radius of the central cluster. The value in each pixel is obtained by summing up the contribution of each gas element along the line of sight in the redshift range $0.65<z<0.33$. The color bar stands for the gas column density in $\mathrm{g} \mathrm{cm}^{-2}$ in $\log$ scale.

We report in Table 1 the median X-ray intensity values (together with the corresponding 15 th and 85th percentiles) obtained for the IGM and the WHIM gas distributions at different wavebands. The analysis of the pixel intensity maps as a function of the median instead of the mean values of the different distributions is less affected by extreme pixel intensities and provides, therefore, a better idea of the 'characteristic' emission. Only in this case, in order to facilitate the comparison with previous works, we show the brightness in units of $\mathrm{erg} \mathrm{s}^{-1} \mathrm{~cm}^{-2} \mathrm{deg}^{-2}$. In doing such a comparison, we need to keep in mind that we only have one large galaxy cluster located in the centre of the simulated box, whereas most of previous studies were based on larger cosmological volumes (e.g. Croft et al. 2001; Phillips et al. 2001; Roncarelli et al. 2006, 2012; Ursino et al. 2014). Despite this issue, the maximum brightness associated to the centre of our galaxy cluster, within $\sim 10^{-10}-10^{-9}$ $\mathrm{erg} \mathrm{s}^{-1} \mathrm{~cm}^{-2} \mathrm{deg}^{-2}$, is in broad agreement with the values associated in cosmological boxes to large galaxy clusters (e.g. Croft et al.
2001; Roncarelli et al. 2006). Moreover, the median values reported in Table 1 also seem to enclose previous independent estimates for the IGM and the WHIM. As an example, in the soft band, Croft et al. (2001) and Roncarelli et al. (2006) reported, respectively, mean brightness values of $\sim 6.58 \times 10^{-12} \mathrm{erg} \mathrm{s}^{-1} \mathrm{~cm}^{-2} \mathrm{deg}^{-2}$ and $\sim 4.06 \times 10^{-12} \mathrm{erg} \mathrm{s}^{-1} \mathrm{~cm}^{-2} \mathrm{deg}^{-2}$ for the IGM and $\sim 4.15 \times$ $10^{-13} \mathrm{erg} \mathrm{s}^{-1} \mathrm{~cm}^{-2} \mathrm{deg}^{-2}$ and $\sim 1.68 \times 10^{-12} \mathrm{erg} \mathrm{s}^{-1} \mathrm{~cm}^{-2} \mathrm{deg}^{-2}$ for the WHIM component. In our case, we obtain a mean emission in the soft band of $6.34 \times 10^{-12} \mathrm{erg} \mathrm{s}^{-1} \mathrm{~cm}^{-2} \mathrm{deg}^{-2}$ for the IGM and $3.18 \times 10^{-13} \mathrm{erg} \mathrm{s}^{-1} \mathrm{~cm}^{-2} \mathrm{deg}^{-2}$ for the WHIM. Despite the different implementations, these values are in line with those previously reported by Croft et al. 2001 (see also Bryan and Voit 2001). In the hard band, our mean intensity values for IGM and WHIM are, respectively, $6.94 \times 10^{-12} \mathrm{erg} \mathrm{s}^{-1} \mathrm{~cm}^{-2} \mathrm{deg}^{-2}$ and $8.68 \times 10^{-14} \mathrm{erg} \mathrm{s}^{-1} \mathrm{~cm}^{-2} \mathrm{deg}^{-2}$, slightly higher than the results obtained by Roncarelli et al. $2006\left((1.01 \pm 1.53) \times 10^{-12}\right.$ $\mathrm{erg} \mathrm{s}^{-1} \mathrm{~cm}^{-2} \mathrm{deg}^{-2}$ and $(2.92 \pm 2.46) \times 10^{-14} \mathrm{erg} \mathrm{s}^{-1} \mathrm{~cm}^{-2} \mathrm{deg}^{-2}$, respectively). From these results we find that, on average, in our simulation the WHIM X-ray emission corresponds to only $\sim 5 \%$ and $\sim 1 \%$ of the IGM emission in the soft and hard X-ray bands, respectively. This relatively small contribution makes even more difficult the detection of the WHIM using X-rays observations.

As for the comparison with observational data, Galeazzi et al. (2009) employed a statistical approach on several XMM-Newton observations to derive a WHIM X-ray contribution to the diffuse $\mathrm{X}$-ray background of $(12 \pm 5) \%$, a value which is roughly consistent with our soft band estimate. On the other hand, the analysis of Chandra deep fields by Hickox and Markevitch (2007) provided an upper limit for the WHIM emission in the $0.65-1 \mathrm{keV}$ energy band of $(1.0 \pm 0.2) \times 10^{-12} \mathrm{erg} \mathrm{s}^{-1} \mathrm{~cm}^{-2} \mathrm{deg}^{-2}$ (see also observational estimates in the soft band by e.g. Briel and Henry 1995; Worsley et al. 2005). Interestingly, and in agreement with previous numerical estimates, our predictions are well-below this value.

In order to highlight and compare the different emissions from the IGM and the WHIM, we show in the right column of Fig. 4 the maps of the hardness ratio (HR) for each gas phase. To maximize the differences, the HR is defined as the ratio of the pixel intensities in the hard $(2-10 \mathrm{keV})$ to the soft $(0.5-2.0 \mathrm{keV})$ energy bands (see e.g. Croft et al. 2001; Roncarelli et al. 2006). In the case of the IGM, the HR map clearly highlights the cluster centre, where the HR values lay above $\sim 0.7-0.8$, reaching the maximum $(\sim 1.18)$ in the densest region. Within the cluster centre and its outskirts there is a region with lower HR values. Regarding the WHIM, its HR map is clearly anti-correlated with that of the IGM, it shows a more extended distribution, and it is characterized by mean HR values smaller by a factor $\sim 9$. Indeed, contrarily to the IGM, the highest HR values in the WHIM map $(\sim 0.065)$ are mostly detected in a thin region close to the cluster limits. In this map, however, any trace of filamentary structures has been almost completely diluted (see also Croft et al. 2001; Roncarelli et al. 2006). Quantitatively, the median HR values that we obtain for the IGM $(\sim 0.01)$ and for the WHIM $(\sim 0.006)$ are slightly below the estimates from Roncarelli et al. (2006), who obtained 0.03 and 0.01 , respectively.

Figure 5 shows the probability distribution functions of the thermal X-ray signal associated to the IGM (blue lines) and to the WHIM (red lines) maps shown in Fig. 4 for the two different energy bands we analyse. For very low intensities $\left(\lesssim 10^{-20}-10^{-19}\right.$ $\mathrm{erg} \mathrm{s}^{-1} \mathrm{~cm}^{-2} \operatorname{arcmin}^{-2}$ ) in a given energy band, associated to very low-temperature gas, IGM and WHIM distributions are very similar. Moreover, most of the pixel maps contribute to this undetectable intensity region. However, as expected, both distributions differ at the high-intensity end, since the high-temperature gas, re- 

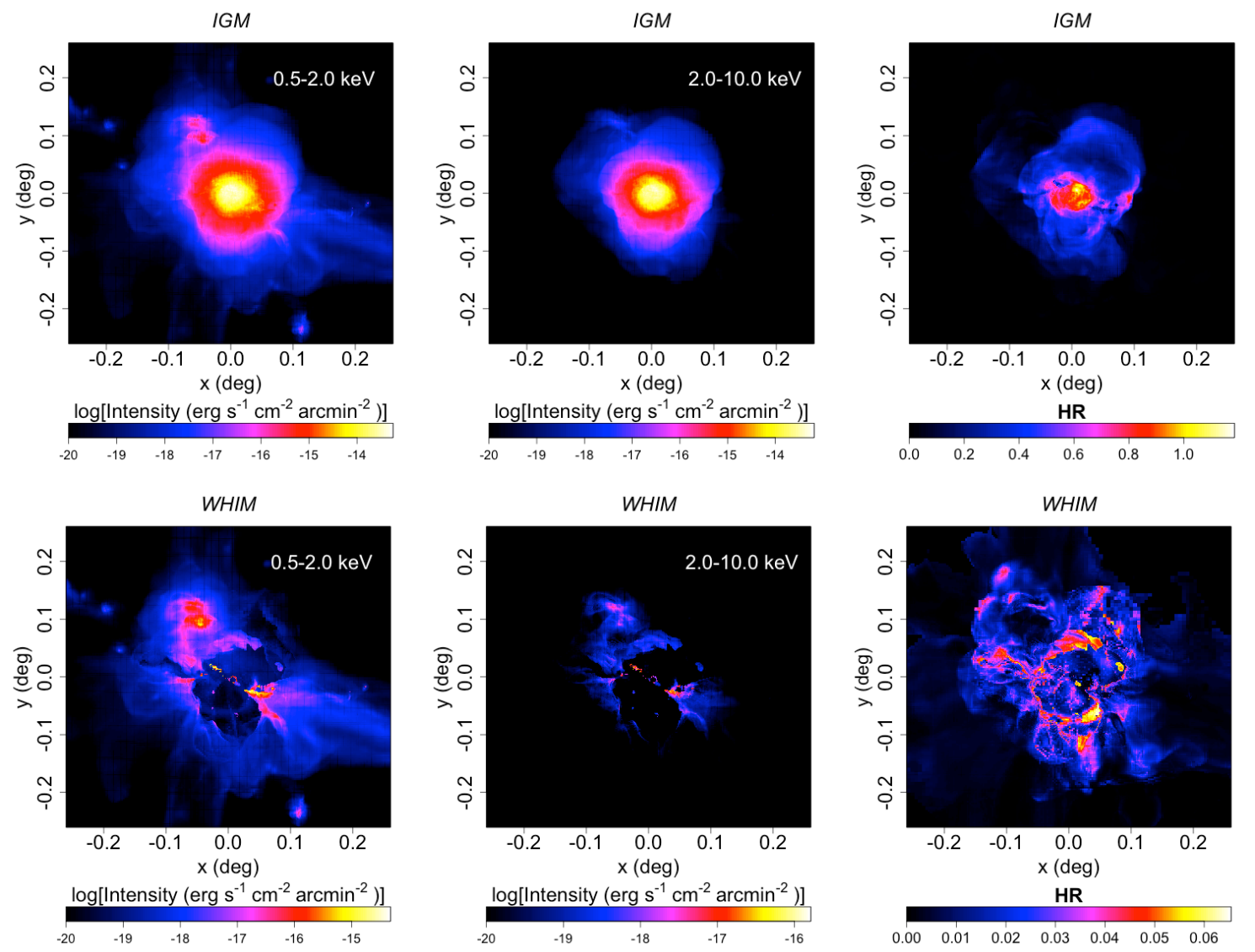

Figure 4. Left and middle columns: X-ray thermal emission maps associated to the IGM and the WHIM gas components (top and bottom rows, respectively) at the soft $(0.5-2 \mathrm{keV})$, and hard $(2-10 \mathrm{keV})$ energy bands. The emission, given in units of $\mathrm{erg} \mathrm{s}^{-1} \mathrm{~cm}^{-2} \operatorname{arcmin}^{-2}$, is plotted in logarithmic scale according to the color code at the bottom of each panel. Each map is sampled with $3200^{2}$ pixels over a region of $\sim 0.6$ squared degrees around the main central halo. Right column: Hardness ratio (HR) maps for the IGM (top panel) and the WHIM (bottom panel) gas components, being HR the ratio of the hard over the soft $\mathrm{X}$-ray emissions.

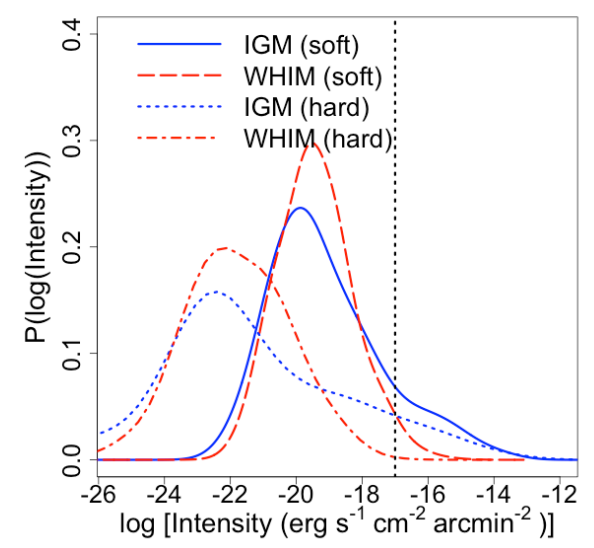

Figure 5. Probability distribution function of the thermal X-ray intensity associated to the all the pixels within the maps shown in Fig. 4. The distributions are shown for the IGM (blue lines) and the WHIM (red lines) in the soft $(0.2-0.5 \mathrm{keV})$ and hard $(2-10 \mathrm{keV}) \mathrm{X}$-ray energy bands. The vertical dotted line represents a threshold detection intensity of $10^{-17}$ $\operatorname{erg~s}{ }^{-1} \mathrm{~cm}^{-2} \operatorname{arcmin}^{-2}$. sponsible of this emission, is included in the IGM but not in the WHIM component. Indeed, in the high-intensity region (above the detection limit marked by the vertical dotted line), the WHIM distribution tends to zero faster than that of the IGM, being almost undetectable in the hard band. Another remarkable fact is that, as already noted by other authors (e.g. Croft et al. 2001; Roncarelli et al. 2006), as we go from the soft to the hard energy band, both distributions become wider, since the number of pixels with null flux increases.

To conclude, we stress that a direct comparison with previous works, either numerical or observational, is far from being straightforward. In this sense, we expect significant discrepancies with other numerical analyses due to not only the use of different box sizes (and, therefore, different cluster catalogues), but also due to the assumption of different cosmological parameter values, the inclusion of different physical processes, the use of different WHIM definitions, or the implementation of different methods to compute the associated X-ray emission (Croft et al. 2001; Bryan and Voit 2001; Roncarelli et al. 2006; Tornatore et al. 2010; Roncarelli et al. 2012; Ursino et al. 2014).

Moreover, we would like to point out that the distribution of metals is sensitive to the treatment of different feedback processes such as galactic winds from SNIa or AGN feedback (e.g. Biffi et al. 2017, 2018). Not accounting for these effects, such as in our cur- 
rent simulation, could thus be a significant source of uncertainties in estimating the X-ray emission from galaxy clusters, as already demonstrated in a number of dedicated studies (e.g. Bertone et al. 2010b; Tornatore et al. 2010; Roncarelli et al. 2012). In general, the inclusion of galactic winds tends to increase the transport of metals from star-forming regions, where they are mainly produced, to outer and less dense IGM regions, producing an increase in the emission from faint lines, especially in the WHIM component (Roncarelli et al. 2012). Contrarily, Roncarelli et al. (2012) also showed that the inclusion of AGN feedback, which tends to prevent the high-redshift collapse of dense regions, produces a reduction in line emission from both IGM and WHIM. On the other hand, as shown by Bertone et al. (2010b), it seems that the distribution of metals in the WHIM is quite stable to different feedback schemes, suggesting that the main point is how the distribution of the gas density is affected by different feedback processes. Therefore, given the complex interplay between different feedback processes and prescriptions, it is difficult to perform a proper comparison between different simulations. However, we would like to highlight that, despite the unavoidable uncertainties and the assumptions adopted in our numerical approach, our results seem to be in a remarkably level of agreement with current X-ray estimates obtained with more complex simulations.

From an observational point of view, we will have to wait for the launch of ATHENA+, by 2028 , to obtain an unprecedented $\mathrm{X}$-ray view of the cosmic web. In comparison with current and planned X-ray facilities, ATHENA + will have a significantly improved spectral $(\sim 2.5 \mathrm{eV})$ and angular $(\sim 5$ arcsec $)$ resolutions, high sensitivity and a large field of view ( $\sim 40 \mathrm{arcmin})$. With these capabilities, it will be able to reveal some of the main cosmic web properties by measuring clusters velocity fields, the group and cluster gas entropy profiles out to high redshifts, and the distribution and evolution of metals. Future observations with ATHENA+ will be also crucial to map the outskirts of clusters, as well as to discover the progenitors of present day galaxy clusters at $z \sim 2.5-3$. Regarding the WHIM, ATHENA+ will be able to detect and measure, with unprecedented precision, the distribution of gas in cosmic filaments through both absorption and emission processes, providing therefore definitive clues on the nature and evolution of the warmhot gas component (see e.g. Nandra et al. 2013; Barcons et al. 2017, for further details).

\subsection{SZ observations}

In this Section we discuss how our cluster is observed via the thermal and kinematic SZ effects. Specifically, as explained in Section 2.3, we compute the total, the thermal and the kinematic SZ contributions to the CMB spectrum at three particular frequencies: $\nu \sim 128 \mathrm{GHz}$, where the tSZ shows its minimum value; $\nu \sim 217 \mathrm{GHz}$, where the tSZ is almost negligible and the spectrum is dominated by the $\mathrm{kSZ}$ signal; and $\nu \sim 369 \mathrm{GHz}$, where the contribution from the tSZ effect to the total spectrum is maximal (e.g. Birkinshaw 1999). Numerical (e.g. Kay et al. 2008; Prokhorov et al. 2010, 2011; Morandi et al. 2013) and observational (e.g. Colafrancesco and Marchegiani 2010) studies have shown that this multi-frequency analysis of the SZ signal represents an extremely useful method to derive the ICM temperature distribution.

Figure 6 shows the intensity maps, $\Delta I / I_{\nu}$, of synthetic SZ observations of our cluster at $\nu=128 \mathrm{GHz}$ for both the IGM and the WHIM gas components (top and bottom rows, respectively). As expected, at this frequency the tSZ map (middle panels) shows always negative values since it produces a reduction in the tempera- ture of the $\mathrm{CMB}$ photons with respect to the $\mathrm{CMB}$ temperature. On the contrary, the kSZ effect (right-hand panels) represents a positive or a negative change in the intensity depending on whether the gas is approaching or going away from the observer. For the sake of completeness, we also show the intensity map associated to the total SZ effect (left-hand panels) computed according to Eq. 9. From the inspection of the tSZ map obtained for the IGM, it is clear that the signal is dominated by the central galaxy cluster, which can reach intensities as high as $\left|\Delta I_{\nu, t h} / I_{\nu}\right| \sim 3 \times 10^{-5}$ in its very central region. Overall, the tSZ signal is broadly distributed in shells of decreasing intensity from the cluster core out to its outskirts. As for the WHIM gas component, most part of the tSZ signal associated to the cluster core is removed, leaving an outer and much fainter signal around the cluster dominated by a more filamentary structure across the central region. If we compute the mean signal from all the pixels contributing to the maps, we obtain $\left|\left\langle\Delta I_{\nu, t h} / I_{\nu}\right\rangle\right| \sim 4 \times 10^{-7}$ for the IGM and $\left|\left\langle\Delta I_{\nu, t h} / I_{\nu}\right\rangle\right| \sim 3 \times 10^{-8}$ for the WHIM component, indicating that most part of the tSZ signal is contributed by hot and/or massive structures. Indeed, the tSZ maps obtained for the IGM and the WHIM at this frequency can be easily correlated with the X-ray thermal emission shown in Fig. 4, especially at the soft X-ray band. Specifically, the brightest regions in X-rays show the largest negative signals in the SZ maps (e.g. Ursino et al. 2014).

On the other hand, when galaxy clusters show bulk motions with respect to the CMB rest frame, SZ observations can be significantly biased by the existence of a significant $\mathrm{kSZ}$ signal. In our case, the comparison of the $\mathrm{kSZ}$ maps associated to the IGM and to the WHIM (right-hand panels of Fig. 6) provides a more similar spatial distribution of their intensities than in the case of the thermal maps. In this case, considering all the pixels contributing to the maps, we obtain mean values of $\left\langle\left|\Delta I_{\nu, k} / I_{\nu}\right|\right\rangle \sim 10^{-9}$ for both the IGM and the WHIM. In general, whereas the gas producing the tSZ signal is also the main contributor to the $\mathrm{X}$-ray emission, the peaks of intensity in the kSZ maps do not always have a clear counterpart in the tSZ maps, since they are mainly associated to small objects with a significant bulk motion (e.g. Roncarelli et al. 2007). Indeed, in this case, most of the kSZ high-intensity regions can be correlated with the smaller subhaloes, the filamentary structure and the mid-overdense regions shown in Fig. 3. Moreover, in comparison with Fig. 4, it is also easy to find their X-ray counterpart in the soft energy band, where the smaller overdensities are highlighted, but not in the hard energy range, where only the highest-density regions are observed. Given the complementarity of the tSZ and $\mathrm{kSZ}$ maps, the total SZ signal shows a more accurate description of both contributions since it highlights both, the signal from highdensity and hot central gas concentrations and the one coming from lower-density and colder small substructures with a non-negligible motion.

As shown in Fig. 7, the qualitative distributions of the different SZ signals at $\nu=369 \mathrm{GHz}$ is very similar to those obtained at $\nu=128 \mathrm{GHz}$ (Fig. 6). The main difference between these two figures is, as expected, in the values of the tSZ maps since, at this higher frequency, the $\mathrm{tSZ}$ effect produces a positive deviation from the CMB spectrum. Moreover, the magnitude of the SZ signal at this high frequency is larger than at lower frequencies. According to the analysis presented in Appendix B, we must take these results with caution since, at $\nu=369 \mathrm{GHz}$, the fact of avoiding the relativistic corrections to compute the SZ effect might have a significant contribution in the final result (see Appendix B for further details).

For the sake of completeness, we provide in Table 2 the median values of the intensity variations, $\Delta I / I_{\nu}$, as obtained from 

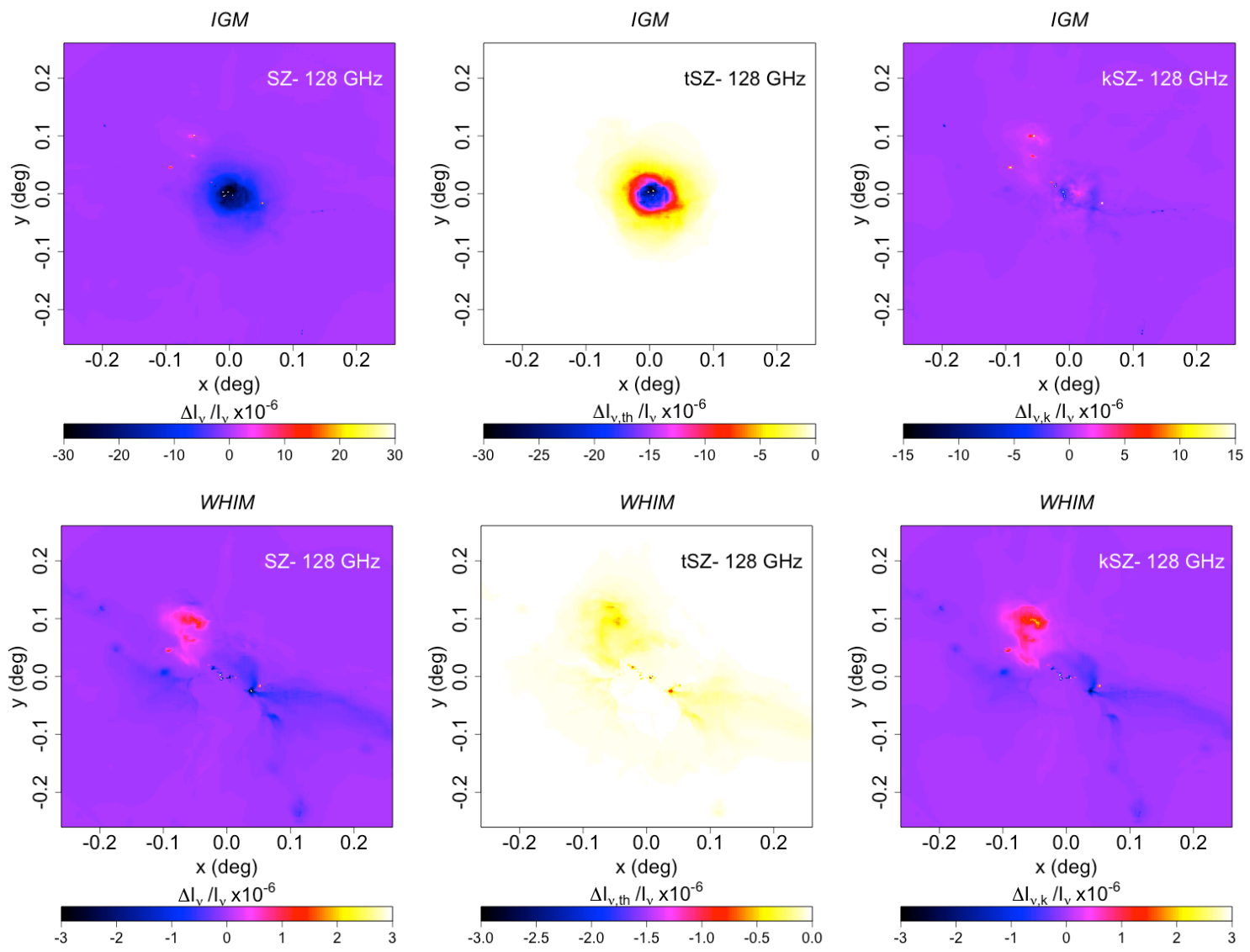

Figure 6. Intensity maps, $\Delta I / I_{\nu}$, of the total, thermal and kinematic SZ effect (panels from left to right, respectively) at a frequency $\nu=128 \mathrm{GHz}$ for the IGM (upper row) and WHIM (bottom row) gas components. Each map is sampled with $1600^{2}$ pixels over a region of $\sim 0.6$ squared degrees around the main central halo, which corresponds to a pixel size of $1.35 \times 1.35 \mathrm{arsec}^{2}$.

\begin{tabular}{cccc}
\hline$\nu(\mathrm{GHz})$ & 128 & 217 & 369 \\
\hline IGM-tSZ & $\left(-2.87_{+1.38}^{-9.19}\right) \times 10^{-6}$ & $\left(-1.30_{+0.18}^{-18.83}\right) \times 10^{-10}$ & $\left(4.63_{+23.40}^{-1.42}\right) \times 10^{-6}$ \\
IGM-kSZ & $\left(-1.04_{+1.66}^{-1.76}\right) \times 10^{-6}$ & $\left(-0.19_{+354.02}^{-69.22}\right) \times 10^{-10}$ & $\left(1.19_{+2.09}^{-2.00}\right) \times 10^{-6}$ \\
WHIM-tSZ & $\left(-1.58_{+1.11}^{-2.63}\right) \times 10^{-6}$ & $\left(-1.19_{+0.19}^{-4.15}\right) \times 10^{-10}$ & $\left(1.33_{+1.99}^{-1.07}\right) \times 10^{-6}$ \\
WHIM-kSZ & $\left(1.19_{+1.52}^{-1.07}\right) \times 10^{-6}$ & $\left(-66.58_{+26.78}^{-62.35}\right) \times 10^{-9}$ & $\left(1.45_{+2.34}^{-1.11}\right) \times 10^{-6}$ \\
\hline
\end{tabular}

Table 2. Median intensity variation values, $\Delta I / I_{\nu}$, of the tSZ and kSZ effects as obtained from the IGM and the WHIM gas components at three different frequencies. Errors represent the 15th and 85 th percentiles of each distribution. We have assumed a frequency-independent threshold intensity variation of $\Delta I / I_{\nu}=10^{-6}$.

the tSZ and kSZ maps associated to the IGM and the WHIM gas components at the three characteristic frequencies we look at. To compute these values we have assumed a frequency-independent threshold intensity variation of $\Delta I / I_{\nu}=10^{-6}$, which is in line with the detection sensitivity of current microwave facilities. In general, as confirmed in previous numerical analyses and observations, the kSZ effect associated to the IGM shows an intensity variation slightly below its corresponding tSZ signal; only at $\nu=217$ $\mathrm{GHz}$, where the tSZ effect has a tiny contribution (several orders below the assumed threshold intensity), both signals are comparable. Moreover, as expected, the WHIM component shows, at all frequencies, a smaller tSZ intensity variation than the one associ- ated to the IGM. However, it is interesting to note that the median $\mathrm{kSZ}$ signal associated to the WHIM is always above the median $\mathrm{kSZ}$ values associated to the IGM. This indicates that, in our selected region, whereas the WHIM (a warm-hot, mildly-overdense intergalactic gas distributed in small substructures and filaments) only has a minor contribution to the total tSZ signal, it dominates the kSZ effect. As far as we are aware of, this is the first comparison between the kSZ signals associated to the IGM and the WHIM. Nevertheless, Nagai et al. (2003) already showed the spatial distribution of the IGM kSZ signal and how this effect could be used to determine cluster peculiar velocities. On average, we find that, at all frequencies, the tSZ signal associated to the WHIM corre- 

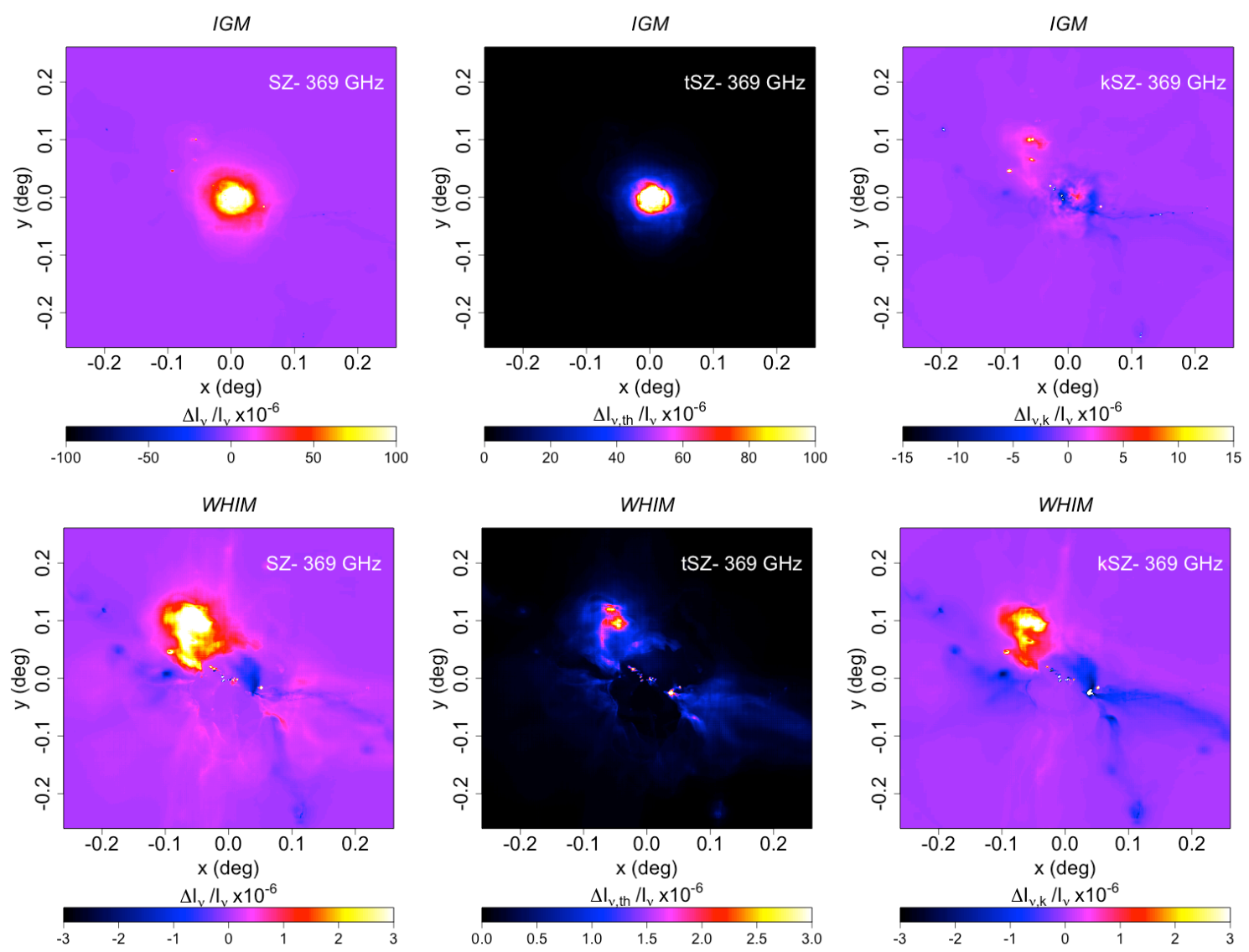

Figure 7. Intensity maps, $\Delta I / I_{\nu}$, of the total, thermal and kinematic SZ effect (panels from left to right, respectively) at a frequency $\nu=369 \mathrm{GHz}$ for the IGM (upper row) and WHIM (bottom row) gas components. These maps have the same resolution than those displayed in Fig. 6.

sponds to only $\sim 6 \%$ of the tSZ signal coming from the IGM, a value that is roughly consistent with the estimation provided by Hallman et al. (2007). In contrast to X-ray observations that show a significant surface-brightness dimming with redshift, SZ observations are redshift-independent. Therefore, despite the weakness of the tSZ signal associated to the WHIM, the detection of this warmhot gas through the SZ effect provides a valuable complementary tool to X-ray observations, especially at high redshift (e.g. Ursino et al. 2014).

In this regard, current multifrequency SZ facilities, such as Planck $^{7}$ (e.g. Planck Collaboration et al. 2011) or $S P T^{8}$ (e.g. Bleem et al. 2015), are detecting a significant number of high-z clusters. Concurrently, a number of additional SZ instruments, such as CCAT-prime $^{9}$ (e.g. Mittal et al. 2017), with higher resolution, sensitivity and spectral coverage, are already planned and will play a crucial role in providing a deeper picture of the ICM and WHIM properties by means of SZ observations alone. Indeed, while there are measurements of the tSZ effect for a large number of clusters (e.g. Bleem et al. 2015), measuring the kinematic (e.g. Sayers et al. 2013) or the relativistic SZ effects (e.g. Zemcov et al. 2012) for individual systems is much more challenging. In this regard, CCATprime will provide a multi-band detection of the SZ effect with improved capabilities. In comparison with Planck, CCAT-prime is

\footnotetext{
7 http://www.esa.int/Our_Activities/Space_Science/Planck

8 https://pole.uchicago.edu

9 http://www.ccatobservatory.org
}

\begin{tabular}{ccc}
\hline $\begin{array}{c}\nu \\
(\mathrm{MHz})\end{array}$ & $\begin{array}{c}\text { IGM } \\
\left(\mu \mathrm{Jy} \mathrm{arsec}^{-2}\right)\end{array}$ & $\begin{array}{c}\text { WHIM } \\
\left(\mu \mathrm{Jy} \mathrm{arsec}^{-2}\right)\end{array}$ \\
\hline 350 (th+nth) & $\left(1.50_{+12.13}^{-0.47}\right) \times 10^{-3}$ & $\left(1.07_{+7.62}^{-0.42}\right) \times 10^{-3}$ \\
1400 (th+nth) & $\left(1.33_{+10.22}^{-0.43}\right) \times 10^{-3}$ & $\left(0.96_{+6.22}^{-0.39}\right) \times 10^{-3}$ \\
1400 (th) & $\left(1.14_{+5.84}^{-0.42}\right) \times 10^{-3}$ & $\left(0.65_{+2.57}^{-0.36}\right) \times 10^{-3}$ \\
\hline
\end{tabular}

Table 3. Median pixel values of the synthetic radio maps shown in Fig. 8 for the IGM and the WHIM gas components at $350 \mathrm{MHz}$ and $1.4 \mathrm{GHz}$ for a beam size of $1 \times 1 \operatorname{arcsec}^{2}$. Errors represent the 15th and 85th percentiles of each distribution. These values are obtained assuming an hypothetical threshold detection intensity of $\sim 3 \times 10^{-4} \mu \mathrm{Jy} \operatorname{arcsec}^{-2}$.

expected to have a much better sensitivity (by an order of magnitude in the $95-405 \mathrm{GHz}$ range) and a much better angular resolution (by a factor $\sim 6$; see Erler et al. 2017, for further details). Moreover, at higher frequency, it will be more efficient in removing point sources. With these capabilities, it will be able to determine individual cluster temperatures and peculiar velocities with an unprecedented precision (see also Morandi et al. 2013, for an analysis of the ICM detection with future CCAT-prime observations). 

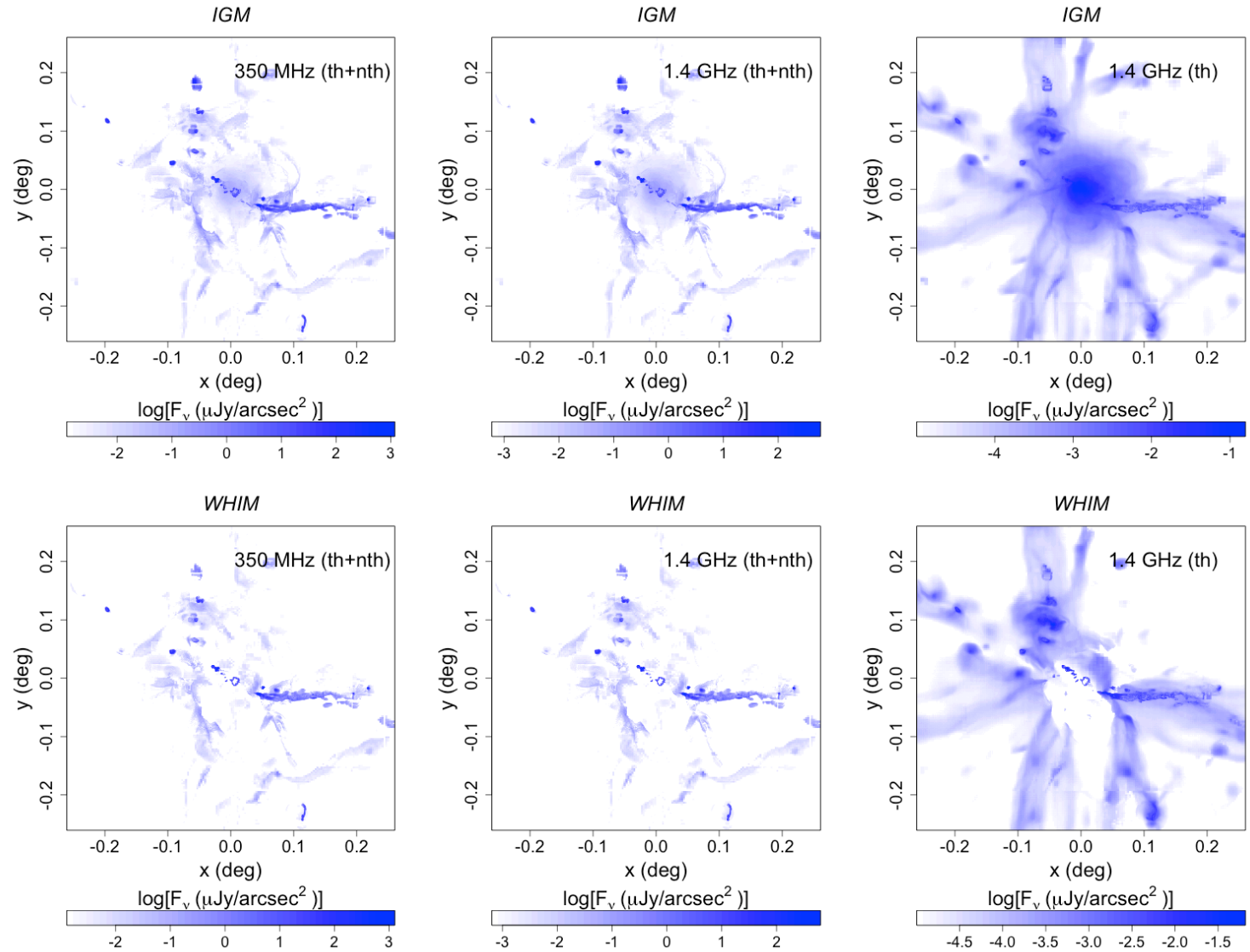

Figure 8. Mock radio observations of the IGM and the WHIM components (top and bottom rows, respectively) at $350 \mathrm{MHz}$ and $1.4 \mathrm{GHz}$ for a beam size of $1 \times 1 \operatorname{arcsec}^{2}$. Left and middle panels show the contribution from the thermal plus non-thermal components $(t h+n t h)$ to the radio maps, whereas the right column shows the subdominant contribution from the thermal component alone.
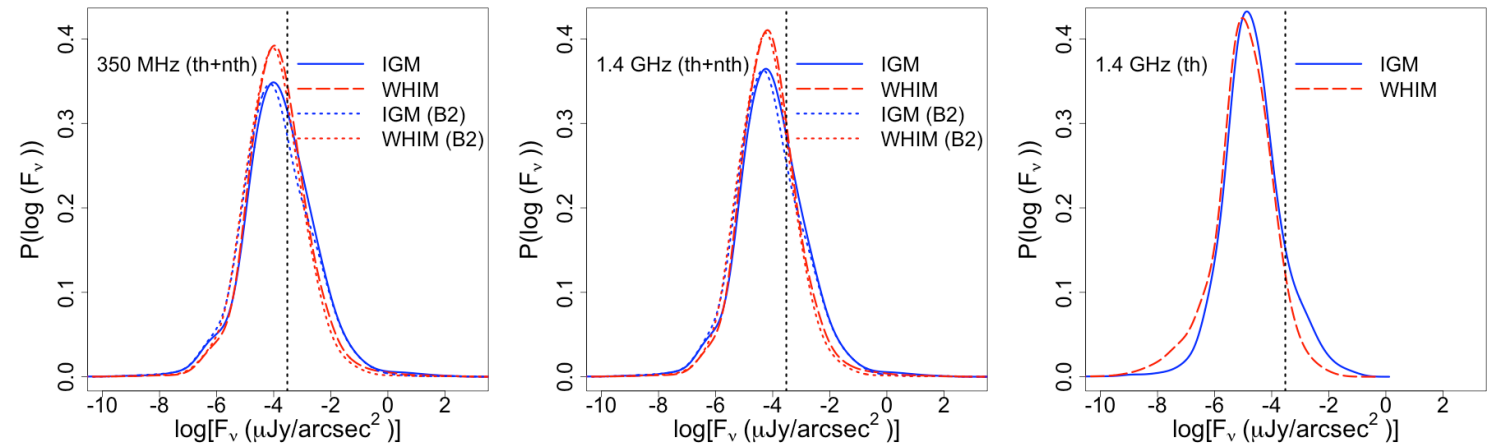

Figure 9. Probability distribution function of the radio signal associated to the pixels composing the maps shown in Fig. 8. The distributions are shown for the IGM (solid blue line) and the WHIM (dashed red line) as contributed by the thermal plus non-thermal components at $350 \mathrm{MHz}$ and $1.4 \mathrm{GHz}$ for a beam size of $1 \times 1 \operatorname{arcsec}^{2}$ (left and middle panels, respectively) and by the thermal component alone at $1.4 \mathrm{GHz}$ (right panel). The vertical dotted line in each panel represents a threshold detection intensity of $1 \mu \mathrm{Jy} \mathrm{arcmin}{ }^{-2}$, which corresponds to $\sim 3 \times 10^{-4} \mu \mathrm{Jy} \operatorname{arcsec}^{-2}$. The distribution of pixel intensities obtained with the simpler magnetic field model provided by Eq. 6 (labelled as model B2) are also shown for comparison.

\subsection{Radio emission}

Radio emission from the cosmic web, connected to merger and accretion structure formation shocks, can also provide independent and complementary information on the existence and evolution of the WHIM (e.g. Brown 2011; Araya-Melo et al. 2012).

As explained in Section 2.2, we have implemented a simplified phenomenological model for shock acceleration in SPEV in order to compute the synchrotron emission in our simulation. We have built synthetic radio maps for the same region around our simulated galaxy cluster as shown in previous sections. We convolve the images with a Gaussian beam whose full width half maximum is $1 \times 1$ $\operatorname{arcsec}^{2}$. In the following, in order to quantify the emission from 


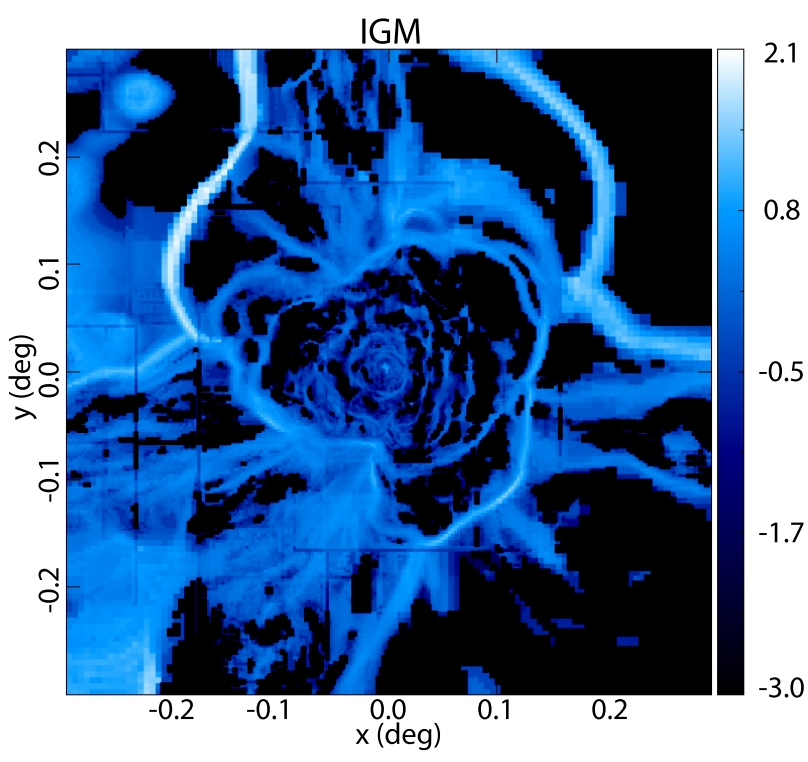

Figure 10. Distribution of shock waves, represented by their shock Mach numbers, within the same region as shown in previous figures around our massive cluster. The map represents a projection along the line of sight for a slice of thickness $0.1 \mathrm{Mpc}$. The color bar shows the Mach number in log scale.

different bands, we will consider a threshold detection intensity of $1 \mu \mathrm{Jy} \operatorname{arcmin}^{-2}$, which corresponds to $\sim 3 \times 10^{-4} \mu \mathrm{Jy} \operatorname{arcsec}^{-2}$. However, we will not adapt our radio maps to the technical properties of any observational instrument.

In particular, the left and middle panels of Fig. 8 show, respectively, the radio emission from the IGM and the WHIM computed at $350 \mathrm{MHz}$ and at $1.4 \mathrm{GHz}$. These images account for the contribution to the radio emission from the thermal and non-thermal gas components $(t h+n t h)$. As expected, we find that only a small fraction of the cluster emits significantly in radio. Moreover, the IGM radio emission is more clearly correlated with high-density and high-temperature regions. Thus, given the features of our emission model, since the density (and, therefore, the magnetic field) and the gas temperature decrease at the cluster periphery, we obtain a very little (if any) radio emission at the very outer cluster regions (around and beyond $\sim 4 \times R_{v i r}$ ). A similar result is also obtained for the WHIM although in this case the emission is distributed in a more filamentary structure, reducing therefore its extension. As obtained in previous simulations, this result is in line with the strong dependence of the radio emission with the cluster temperature (e.g. Hoeft et al. 2008). However, although the radio emission associated to the WHIM is less extended, at $350 \mathrm{MHz}$, the mean radio signals associated to the WHIM $\left(\sim 0.55 \mu \mathrm{Jy} \operatorname{arcsec}^{-2}\right)$ and to the whole IGM $\left(\sim 0.63 \mu \mathrm{Jy} \operatorname{arcsec}^{-2}\right)$ are very similar. This is obviously due to the fact that, when computing the mean pixel intensity, the high-intensity pixels have more weight than the low-intensity ones in the WHIM maps. If we look instead to the median pixel values reported in Table 3, the radio signal associated to the IGM is larger than the one associated to the WHIM by a factor $\sim 1.4$. Moreover, when we go from $350 \mathrm{MHz}$ to $1.4 \mathrm{GHz}$, we obtain a $\sim 60 \%$ fainter, but a slightly more spatially distributed, mean radio emission from both the IGM and the WHIM components. Overall, at $1.4 \mathrm{GHz}$, we obtain for IGM and WHIM a maximum radio signal of half mJy $\operatorname{arcmin}^{-2}$, whereas at $350 \mathrm{MHz}$ the maximum emission is of the order of $\sim 1-2 \mathrm{mJy} \operatorname{arcmin}^{-2}$.

We can compare these results, obtained with our magnetic field reference model given by Eq. 2, with those obtained with the simpler B2 model provided by Eq. 6 . In this case we obtain, at both considered frequencies, an increase of a factor $\sim 2$ and $\sim 3$ for the mean emissions from the IGM and the WHIM, respectively. Moreover, the maximum emissions associated to the IGM and to the WHIM are also increased by a factor $\sim 3$ and $\sim 5$, respectively. Given the characteristics of our simulation and the approximations adopted in our post-processing, we should take with caution our results. Nevertheless, we can accept as an upper limit the results on the radio emission obtained with the magnetic field model B2 provided by Eq. 6 .

For the sake of completeness, the right column of Fig. 8 shows the contribution of the thermal component alone to the radio maps at $1.4 \mathrm{GHz}$. As expected, the thermal contribution is highly correlated with the density maps shown in Fig. 3 and its global contribution to the radio signal is significantly smaller (by a factor $\sim 4$ ) than the non-thermal contribution.

Despite the clear difference in the emission level, Fig. 9 shows that the probability distribution functions of the radio signal associated to the pixels composing the radio maps of Fig. 8 display a similar gaussian-like distribution, with the IGM dominating over the WHIM radio emission at the high-flux end of the distributions at both frequencies. A similar conclusion is obtained if we compare the pixel intensity distributions resulting from the reference and the B2 magnetic field models. As expected, these two models only affect the non-thermal emission and, therefore, they generate an identical thermal distribution at $1.4 \mathrm{GHz}$ (right panel of Fig. 9).

To complement these radio maps, Fig. 10 shows the distribution of shock waves within our region of interest for a thin slice of $\sim 0.1 \mathrm{Mpc}$ along the line of sight. In particular, shock waves are represented by their Mach numbers, which give an idea of the shock strength. From this distribution, it is easy to distinguish internal, low-Mach number shocks $(\mathcal{M}<10)$ from more external and stronger shocks $(\mathcal{M}>10)$ mainly wrapping the central region. Although Fig. 8 and Fig. 10 are not directly comparable, they suggest that, in our case, most of the cluster radio emission is associated to non-thermal internal shock events within the cluster, whereas radio emission connected to external accretion shocks is negligible (see also Hoeft et al. 2008; Araya-Melo et al. 2012). It has been suggested that the radio emission associated to large-scale accretion shocks could be detected by the next generation of radio telescopes, such as the Low Frequency Array ${ }^{10}$ (LOFAR; e.g. Röttgering 2003) or the Square-Kilometre Array ${ }^{11}$ (SKA; e.g. Keshet et al. 2004; Acosta-Pulido et al. 2015). However, the results of our analysis on the radio band suggest that such a low emission from the accretion region $\left(\sim 4 \times R_{v i r}\right)$ would be undetectable even with future radio facilities.

Observationally, the detection of the WHIM radio emission is extremely difficult. Previous simulations (e.g. Pfrommer et al. 2008; Battaglia et al. 2009), including a complex treatment for the physics of the non-thermal electron population, predict a maximum level of radio emission in the outskirts of massive clusters of only a few $\sim \mu \mathrm{Jy} \operatorname{arcmin}^{-2}$ at $1.4 \mathrm{GHz}$. Moreover, radio observations at high frequencies also suffer contamination from large spatial scales and confusion sources (e.g. Brown 2011; Clarke et al. 2014). In-

\footnotetext{
10 https://www.lofar.org/

11 https://www.skatelescope.org/
} 
deed, above $1 \mathrm{GHz}$, the detection of shock structures through future polarization observations seems to be the most hopeful method to detect the radio emission associated to the WHIM. In addition, later analyses have also suggested that the next generation of radio telescopes will be able to detect the cosmic web at lower frequencies (<200 MHz; e.g. Vazza et al. 2015b,a, 2016). In this regard, the $S K A$ will allow observations of the cosmic web with unprecedented resolution and sensitivity over a large range of frequencies $(\sim 0.05-20 \mathrm{GHz}$; Kale et al. 2016) and beyond $z=0.4$. It will be able to detect lower surface brightness (by a factor $10-50$ than current facilities) and extended cluster radio emission, thus allowing the detection of a significant number of clusters (as an example, it is expected to discover thousands of new relics, radio haloes and mini haloes with a typical emission of $\sim 0.2-1 \mu \mathrm{Jy} \operatorname{arcsec}^{-2}$ at $1.4 \mathrm{GHz}$ ). Regarding the WHIM, as predicted by Araya-Melo et al. (2012), if electrons are accelerated by accretion shocks, radio fluxes of $\sim 0.12 \mu \mathrm{Jy}$ at $150 \mathrm{MHz}$ from filaments at $z=0.15$ will be detected. This will also allow to put constraints on the amount of magnetic fields and cosmic rays in filaments.

\section{SUMMARY AND CONCLUSIONS}

The combination of multi-wavelength observations of the cosmic web is crucial to deepen our knowledge of the Universe in a number of aspects, such as the physics of baryons, the magnetization of the IGM, the pressure of the hot ICM gas or the formation and evolution of cosmic shock waves. Therefore, in view of improved and upcoming observational facilities, such as ATHENA+, the $S K A$ or the CCAT-prime, it is crucial to analyse multi-wavelength synthetic observations performed with full cosmological simulations.

In this paper, we analyse a massive galaxy cluster formed and evolved in an Eulerian-AMR cosmological simulation in order to investigate the spatial distribution and the emission associated to the WHIM gas component, defined as the gas with a temperature in the range $10^{5}-10^{7} \mathrm{~K}$. A set of multi-wavelength synthetic observations around the largest galaxy cluster $\left(M_{v i r} \sim 3.2 \times 10^{14} M_{\odot}\right.$ and $R_{v i r} \sim 1.7 \mathrm{Mpc}$ ) developed in our simulation is built. To do so, we present a novel numerical methodology in which we apply the relativistic full-radiative transfer code SPEV (Mimica et al. 2009; Cuesta-Martínez et al. 2015; Mimica et al. 2016) on the outcomes of the simulation in order to compute the emission associated to both the whole IGM and the WHIM gas components. The upgrade version of SPEV computes the intensity along each line of sight by integrating the transfer equation along the null-geodesic of photons. The emission in the three studied bands, namely, X-ray, SZ, and radio, is computed using exactly the same numerical scheme and, therefore, all bands are consistently treated. In order to study the different spatial distributions of both gas components, we consider a region of $5 \times R_{v i r}$ around our central system. This choice allows us to produce detailed synthetic emission maps of both gas phases, from the cluster core out to a very external region covering an angular extension of $\sim 0.6$ squared degrees.

Besides the presentation of our numerical procedure, the main results obtained in the current analysis on the emission properties of the IGM and the WHIM can be summarised as follows:

- The z-evolution of different IGM gas components within the whole $(40 \mathrm{Mpc})$ simulated box shows that most of the volume ( $90 \%)$ is dominated by the warm $\left(T<10^{5} \mathrm{~K}\right)$ gas phase, whereas the WHIM $\left(10^{5} \mathrm{~K}<\mathrm{T}<10^{7} \mathrm{~K}\right)$ and the hot $\left(T>10^{7} \mathrm{~K}\right)$ gas components occupy, respectively, $\sim 10 \%$ and a few per cent of the domain.
- As evolution proceeds and the largest cosmic structures begin to be formed, the amount of gas in the hot phase increases from $10 \%$ at $z=1$ up to $20 \%$ at $z=0$. Concurrently, while the amount of gas in the warm component slightly decreases down to $30 \%$ at $z=0$, the amount of WHIM mildly augments from $50 \%$ at $z=1$ up to a value of $\sim 55 \%$ at the present epoch (in line with previous numerical analyses; e.g. Cen and Ostriker 1999b).

- The density maps obtained for the whole IGM and for the WHIM reveal two different distributions: while in the former, highdensity regions are clearly connected with the largest haloes and subhaloes in the simulation, the WHIM tends to reside around the main central halo with a more filamentary structure that extends out to more external regions.

- On average, we obtain that the WHIM X-ray emission corresponds to $\sim 5 \%$ and $1 \%$ of the IGM emission in the soft $(0.5-2$ $\mathrm{keV})$ and hard $(2-10 \mathrm{keV}) \mathrm{X}$-ray bands, respectively. This makes the detection of the WHIM a challenge for current observational instruments.

- The maximum values of the X-ray surface brightness in the centre of our galaxy cluster $\left(\sim 10^{-11}-10^{-9} \mathrm{erg} \mathrm{s}^{-1} \mathrm{~cm}^{-2} \mathrm{deg}^{-2}\right)$ are in broad agreement with previous estimates (e.g. Croft et al. 2001; Roncarelli et al. 2006).

- The tSZ maps obtained for the IGM and for the WHIM at $\nu=$ $128 \mathrm{GHz}$ can be easily correlated with the X-ray thermal emission of both components, especially at the soft X-ray band. Specifically, the brightest regions in X-rays show the largest negative signals in the SZ maps (e.g. Ursino et al. 2014). We obtain intensities as high as $\left|\Delta I_{\nu, t h} / I_{\nu}\right| \sim 3 \times 10^{-5}$ in the very central cluster region.

- The kSZ maps associated to the IGM and to the WHIM show a more similar spatial distribution of their intensities than in the case of the thermal maps. In this case, we obtain mean values of $\left\langle\left|\Delta I_{\nu, k} / I_{\nu}\right|\right\rangle \sim 10^{-9}$ for both gas components (see also Nagai et al. 2003).

- Qualitatively, the distribution of the different SZ signals at $\nu=369 \mathrm{GHz}$ is very similar to those obtained at $\nu=128$ GHz. However, our results at this high frequency, obtained in the non-relativistic approximation, must be taken with caution. Indeed, through a comparison with the SZ signal obtained with SZPACK (Chluba et al. 2012, 2013), we have confirmed that avoiding the relativistic corrections at $\nu=369 \mathrm{GHz}$ can significantly affect our estimations, especially for the IGM.

- On average, we find that, at all frequencies, the tSZ signal associated to the WHIM corresponds to $\sim 6 \%$ of the tSZ signal from the IGM (roughly consistent with the estimate by Hallman et al. 2007).

- We find that only a small fraction of the cluster emits in radio. In addition, whereas the IGM radio emission is quite correlated with high-density and high-temperature regions, the radio emission associated to the WHIM is distributed in a more filamentary structure.

- On average, at $1.4 \mathrm{GHz}$ we obtain a radio signal $\sim 60 \%$ fainter than at $350 \mathrm{MHz}$ for both the IGM and the WHIM. As a mean upper limit for the radio signal at $1.4 \mathrm{GHz}(350 \mathrm{MHz})$ we get a value of half $\mathrm{mJy} \operatorname{arcmin}^{-2}\left(1-2 \mathrm{mJy} \operatorname{arcmin}^{-2}\right)$ for the IGM and the WHIM.

- At both radio frequencies, $350 \mathrm{MHz}$ and $1.4 \mathrm{GHZ}$, the cluster radio emission seems to be mainly associated to non-thermal internal shock events within the cluster. Contrarily, we obtain a negligible radio emission connected to external accretion shocks (see also Hoeft et al. 2008; Araya-Melo et al. 2012).

We would like to point out that the results presented in this pa- 
per should be taken as our first step towards designing a technique that allows us to treat, consistently, the IGM emission at any frequency. To fulfill this objective, we use a full-radiative transfer code that implies no approximations nor simplifications, as the transfer equation is solved along the null-geodesic of photons. Nevertheless, despite this ambitious intention, we are aware of the current limitations of our simulation and of our numerical procedure. In this regard, a self-consistent treatment of the metal distribution, a proper modeling of fundamental feedback processes, such as AGN feedback or galactic winds, or the inclusion of the relativistic corrections to estimate the SZ effect represent significant improvements to be accounted for. Having in mind these limitations, we chose as a first target for our study a very high-resolution galaxy cluster $(\sim 610 \mathrm{pc})$, in contrast with previous numerical approaches which were focused on larger but lower resolution cluster samples. The idea behind this choice was to show the capability of SPEV to compute multi-wavelength synthetic observations and to produce well-resolved mock maps in order to facilitate the comparison with the imminent observational data arriving from forthcoming facilities. Now that the method has been fully tested and is fully functional, we are working on a new set of simulations that, while keeping a very high resolution, will produce a large catalogue of clusters. Undoubtedly, a major step forward in our understanding of the nature and the role of gas in cosmic structures will come from the combined analysis of multi-wavelength observations produced by the next generation of telescopes, together with mock observations derived from high-resolution cosmological simulations including all relevant physical processes. In this regard, the unprecedented observational capabilities of upcoming X-ray, radio and submillimeter observatories, such as ATHENA+, SKA or CCAT-prime, are expected to provide the most valuable conclusions on the main properties of the IGM and the WHIM gas components.

\section{ACKNOWLEDGEMENTS}

Authors would like to thank Jens Chluba for valuable discussions and his help in running the SZPACK code as well as the anonymous referee for his/her constructive criticism. We acknowledge support by the Spanish Ministerio de Economía y Competitividad (MINECO, grant AYA2016-77237-C3-3-P) and the Generalitat Valenciana (grant GVACOMP2015-227). SP is "Juan de la Cierva" fellow (ref. IJCI-2015-26656) of the Spanish MINECO. PM acknowledges the support from the European Research Council (grant CAMAP-259276). CC-M acknowledges the support of ACIF/2013/278 fellowship. We acknowledge the use of publicly available $\mathrm{R}$ packages $\mathrm{tidy} r$ and $\mathrm{dp} \mathrm{ly}_{\mathrm{y} r}{ }^{12}$ for data manipulation and ggplot $2^{13}$ for producing graphics as well as the VisIt visualisation software (Childs et al. 2012).

\section{REFERENCES}

Acosta-Pulido J.A., et al., 2015, ArXiv e-prints

Araya-Melo P.A., Aragón-Calvo M.A., Brüggen M., Hoeft M., 2012, MNRAS, 423, 2325

Barcons X., et al., 2017, Astronomische Nachrichten, 338, 153

Basu K., Erler J., Sommer M., Vazza F., Eckert D., 2016, Galaxies, 4, 73

12 http://https://www.tidyverse.org/

13 https://www.ggplot2.org/
Battaglia N., Pfrommer C., Sievers J.L., Bond J.R., Enßlin T.A., 2009, MNRAS, 393, 1073

Bertone S., Schaye J., Dolag K., 2008, Space Sci. Rev., 134, 295

Bertone S., Schaye J., Booth C.M., Dalla Vecchia C., Theuns T., Wiersma R.P.C., 2010a, MNRAS, 408, 1120

Bertone S., Schaye J., Dalla Vecchia C., Booth C.M., Theuns T., Wiersma R.P.C., 2010b, MNRAS, 407, 544

Biffi V., Planelles S., Borgani S., Rasia E., Murante G., Fabjan D., Gaspari M., 2018, ArXiv e-prints

Biffi V., et al., 2017, MNRAS, 468, 531

Birkinshaw M., 1999, Phys. Rep., 310, 97

Bleem L.E., et al., 2015, ApJS, 216, 27

Böhringer H., Werner N., 2010, A\&ARv, 18, 127

Branchini E., et al., 2009, ApJ, 697, 328

Bregman J.N., 2007, ARA\&A, 45, 221

Briel U.G., Henry J.P., 1995, A\&A, 302, L9

Brown S.D., 2011, Journal of Astrophysics and Astronomy, 32, 577

Brunetti G., Jones T.W., 2014, International Journal of Modern Physics D, 23, 1430007-98

Bryan G.L., Norman M.L., 1998, ApJ, 495, 80

Bryan G.L., Voit G.M., 2001, ApJ, 556, 590

Carlstrom J.E., Holder G.P., Reese E.D., 2002, ARA\&A, 40, 643

Cen R., Fang T., 2006, ApJ, 650, 573

Cen R., Ostriker J.P., 1999a, ApJ, 519, L109

Cen R., Ostriker J.P., 1999b, ApJ, 514, 1

Cen R., Ostriker J.P., 2006, ApJ, 650, 560

Childs H., et al., 2012, in High Performance Visualization-Enabling Extreme-Scale Scientific Insight, pp. 357-372

Chluba J., Nagai D., Sazonov S., Nelson K., 2012, MNRAS, 426, 510

Chluba J., Switzer E., Nelson K., Nagai D., 2013, MNRAS, 430, 3054

Clarke T., et al., 2014, ArXiv e-prints

Colafrancesco S., 2007, New Astron. Rev., 51, 394

Colafrancesco S., Marchegiani P., 2010, A\&A, 520, A31

Croft R.A.C., Di Matteo T., Davé R., Hernquist L., Katz N., Fardal M.A., Weinberg D.H., 2001, ApJ, 557, 67

Cuesta-Martínez C., Aloy M.A., Mimica P., 2015, MNRAS, 446, 1716

Davé R., et al., 2001, ApJ, 552, 473

Dolag K., Bykov A.M., Diaferio A., 2008, Space Sci. Rev., 134, 311

Eckert D., Produit N., Paltani S., Neronov A., Courvoisier T.J.L., 2008, A\&A, 479, 27

Eckert D., et al., 2015, Nature, 528, 105

Eisenstein D.J., Hu W., 1998, ApJ, 496, 605

Enßlin T.A., Pfrommer C., Springel V., Jubelgas M., 2007, A\&A, 473, 41

Erler J., Basu K., Chluba J., Bertoldi F., 2017, ArXiv e-prints

Fang T., Canizares C.R., Yao Y., 2007, ApJ, 670, 992

Feretti L., Giovannini G., 2008, in M. Plionis, O. López-Cruz, D. Hughes, eds., A Pan-Chromatic View of Clusters of Galaxies and the LargeScale Structure, vol. 740 of Lecture Notes in Physics, Berlin Springer Verlag, p. 24

Ferland G.J., et al., 2017, Rev. Mex. Astron. Astrofis., 53, 385

Ferrari C., Govoni F., Schindler S., Bykov A.M., Rephaeli Y., 2008, Space Sci. Rev., 134, 93

Finoguenov A., Briel U.G., Henry J.P., 2003, A\&A, 410, 777

Fixsen D.J., 2009, ApJ, 707, 916

Fukugita M., Hogan C.J., Peebles P.J.E., 1998, ApJ, 503, 518

Fusco-Femiano R., Orlandini M., Brunetti G., Feretti L., Giovannini G., Grandi P., Setti G., 2004, ApJ, 602, L73

Galeazzi M., Gupta A., Ursino E., 2009, ApJ, 695, 1127

Gheller C., Vazza F., Favre J., Brüggen M., 2015, MNRAS, 453, 1164

Haardt F., Madau P., 1996, ApJ, 461, 20

Hallman E.J., O’Shea B.W., Burns J.O., Norman M.L., Harkness R., Wagner R., 2007, ApJ, 671, 27

Hernández-Monteagudo C., Trac H., Verde L., Jimenez R., 2006, ApJ, 652, L1

Hernández-Monteagudo C., Ma Y.Z., Kitaura F.S., Wang W., GénovaSantos R., Macías-Pérez J., Herranz D., 2015, Physical Review Letters, 115,191301

Hickox R.C., Markevitch M., 2007, ApJ, 661, L117 
Hoeft M., Brüggen M., Yepes G., Gottlöber S., Schwope A., 2008, MNRAS, 391, 1511

Hoffman Y., Ribak E., 1991, ApJ, 380, L5

Itoh N., Kohyama Y., Nozawa S., 1998, ApJ, 502, 7

Kale R., et al., 2016, Journal of Astrophysics and Astronomy, 37, 31

Katz N., Weinberg D.H., Hernquist L., 1996, ApJS, 105, 19

Kay S.T., Powell L.C., Liddle A.R., Thomas P.A., 2008, MNRAS, 386, 2110

Keshet U., Waxman E., Loeb A., 2004, ApJ, 617, 281

Kirkman D., Tytler D., Suzuki N., O'Meara J.M., Lubin D., 2003, ApJS, 149,1

Knebe A., et al., 2011, MNRAS, 415, 2293

Kravtsov A.V., Borgani S., 2012, ARA\&A, 50, 353

Kravtsov A.V., Klypin A., Hoffman Y., 2002, ApJ, 571, 563

Lufkin E.A., Sarazin C.L., White III R.E., 2000, ApJ, 542, 94

Martin-Alvarez S., Planelles S., Quilis V., 2017, Ap\&SS, 362, 91

Mimica P., Aloy M.A., Agudo I., Martí J.M., Gómez J.L., Miralles J.A., 2009, ApJ, 696, 1142

Mimica P., Aloy M.A., Giannios D., Metzger B.D., 2016, Journal of Physics Conference Series, 719, 012008

Miniati F., 2001, Computer Physics Communications, 141, 17

Mittal A., de Bernardis F., Niemack M.D., 2017, ArXiv e-prints

Molendi S., Gastaldello F., 2009, A\&A, 493, 13

Morandi A., Nagai D., Cui W., 2013, MNRAS, 431, 1240

Nagai D., Kravtsov A.V., Kosowsky A., 2003, ApJ, 587, 524

Nandra K., et al., 2013, ArXiv e-prints

Navarro-González J., Ricciardelli E., Quilis V., Vazdekis A., 2013, MNRAS, 436, 3507

Nicastro F., Krongold Y., Fields D., Conciatore M.L., Zappacosta L., Elvis M., Mathur S., Papadakis I., 2010, ApJ, 715, 854

Nicastro F., et al., 2013, ApJ, 769, 90

Nozawa S., Itoh N., Kohyama Y., 2005, A\&A, 440, 39

Paerels F., Kaastra J., Ohashi T., Richter P., Bykov A., Nevalainen J., 2008, Space Sci. Rev., 134, 405

Pérez-Torres M.A., Zandanel F., Guerrero M.A., Pal S., Profumo S., Prada F., Panessa F., 2009, MNRAS, 396, 2237

Pfrommer C., Enßlin T.A., Springel V., 2008, MNRAS, 385, 1211

Phillips L.A., Ostriker J.P., Cen R., 2001, ApJ, 554, L9

Planck Collaboration, et al., 2011, A\&A, 536, A8

Planck Collaboration, et al., 2013, A\&A, 550, A134

Planck Collaboration, et al., 2016, A\&A, 594, A13

Planelles S., Quilis V., 2010, A\&A, 519, A94

Planelles S., Quilis V., 2013, MNRAS, 428, 1643

Planelles S., Borgani S., Dolag K., Ettori S., Fabjan D., Murante G., Tornatore L., 2013, MNRAS, 431, 1487

Planelles S., Schleicher D.R.G., Bykov A.M., 2015, Space Sci. Rev., 188, 93

Prokhorov D.A., Dubois Y., Nagataki S., 2010, A\&A, 524, A89

Prokhorov D.A., Dubois Y., Nagataki S., Akahori T., Yoshikawa K., 2011, MNRAS, 415, 2505

Quilis V., 2004, MNRAS, 352, 1426

Quilis V., Planelles S., Ricciardelli E., 2017, ArXiv e-prints

Rauch M., 1998, ARA\&A, 36, 267

Reiprich T.H., Basu K., Ettori S., Israel H., Lovisari L., Molendi S., Pointecouteau E., Roncarelli M., 2013, Space Sci. Rev., 177, 195

Rephaeli Y., Nevalainen J., Ohashi T., Bykov A.M., 2008, Space Sci. Rev., 134,71

Richter P., Paerels F.B.S., Kaastra J.S., 2008, Space Sci. Rev., 134, 25

Roncarelli M., Moscardini L., Tozzi P., Borgani S., Cheng L.M., Diaferio A., Dolag K., Murante G., 2006, MNRAS, 368, 74

Roncarelli M., Moscardini L., Borgani S., Dolag K., 2007, MNRAS, 378, 1259

Roncarelli M., Cappelluti N., Borgani S., Branchini E., Moscardini L., 2012, MNRAS, 424, 1012

Röttgering H., 2003, New Astron. Rev., 47, 405

Rybicki G.B., Lightman A.P., 1979, Radiative processes in astrophysics, Wiley-Interscience, New York, rL79
Sanders J.S., Fabian A.C., Allen S.W., Schmidt R.W., 2004, MNRAS, 349, 952

Sanders J.S., Fabian A.C., Dunn R.J.H., 2005, MNRAS, 360, 133

Sayers J., et al., 2013, ApJ, 778, 52

Scharf C., Donahue M., Voit G.M., Rosati P., Postman M., 2000, ApJ, 528, L73

Shull J.M., Smith B.D., Danforth C.W., 2012, ApJ, 759, 23

Springel V., Hernquist L., 2003, MNRAS, 339, 289

Sunyaev R.A., Zel'dovich I.B., 1980, MNRAS, 190, 413

Sunyaev R.A., Zel'dovich Y.B., 1972, Comments on Astrophysics and Space Physics, 4, 173

Sutherland R.S., Dopita M.A., 1993, ApJS, 88, 253

Takei Y., et al., 2011, ApJ, 734, 91

Theuns T., Leonard A., Efstathiou G., Pearce F.R., Thomas P.A., 1998, MNRAS, 301, 478

Tornatore L., Borgani S., Viel M., Springel V., 2010, MNRAS, 402, 1911

Ursino E., Galeazzi M., 2006, ApJ, 652, 1085

Ursino E., Galeazzi M., Huffenberger K., 2014, ApJ, 789, 55

van Hoof P.A.M., Williams R.J.R., Volk K., Chatzikos M., Ferland G.J., Lykins M., Porter R.L., Wang Y., 2014, MNRAS, 444, 420

van Hoof P.A.M., Ferland G.J., Williams R.J.R., Volk K., Chatzikos M., Lykins M., Porter R.L., 2015, MNRAS, 449, 2112

Vazza F., Ferrari C., Bonafede A., Brüggen M., Gheller C., Braun R., Brown S., 2015a, Advancing Astrophysics with the Square Kilometre Array (AASKA14), 97

Vazza F., Ferrari C., Brüggen M., Bonafede A., Gheller C., Wang P., 2015b, A\&A, 580, A119

Vazza F., Brueggen M., Gheller C., Ferrari C., Bonafede A., 2016, ArXiv e-prints

Vazza F., Brüggen M., Gheller C., Hackstein S., Wittor D., Hinz P.M., 2017, Classical and Quantum Gravity, 34, 234001

Vazza F., Brunetti G., Brüggen M., Bonafede A., 2018, MNRAS, 474, 1672

Werner N., Finoguenov A., Kaastra J.S., Simionescu A., Dietrich J.P., Vink J., Böhringer H., 2008, A\&A, 482, L29

Worsley M.A., et al., 2005, MNRAS, 357, 1281

Yepes G., Kates R., Khokhlov A., Klypin A., 1997, MNRAS, 284, 235

Zappacosta L., Nicastro F., Maiolino R., Tagliaferri G., Buote D.A., Fang T., Humphrey P.J., Gastaldello F., 2010, ApJ, 717, 74

Zemcov M., et al., 2012, ApJ, 749, 114

\section{APPENDIX A: COMPUTING THERMAL X-RAY EMISSION USING CLOUDY}

We use the publicly available code CLOUDY (version 17.00; Ferland et al. 2017) to construct a large interpolation table of the Xray emission in the soft and hard bands. In this section we give details about the computation, as well as comparisons with thermal Bremsstrahlung and the interpolation errors.

\section{A1 Interpolation table}

To compute the X-ray images in this work we need to be able to model the plasma emission in a large dynamic range of parameters. To achieve this we used CLOUDY to compute a grid of models with the following axes:

- temperature $T$ : 51 logarithmically spaced nodes between $10^{3.6} \mathrm{~K}$ and $10^{8.6} \mathrm{~K}$,

- hydrogen density $n_{H}$ : 131 logarithmically spaced nodes between $10^{-7.7} \mathrm{~cm}^{-3}$ and $10^{5.3} \mathrm{~cm}^{-3}$,

- logarithm of metallicity relative to solar $\log \left(Z / Z_{\odot}\right): 17$ points uniformly spaced between -2.2 and -0.6 ,

- redshift $z$ : since we know which simulation snapshots can possibly contribute to the emission (see Section 2.4), to reduce the table computing time we use their redshifts instead of a denser grid; 
the 6 redshifts are $0.3516,0.3821,0.4126,0.4429,0.4732$ and 0.5051 ,

- $\mathcal{D}_{Z}$, not used by CLOUDY, but used to construct the final table: a combination of the Doppler factor and the redshift $\left(\mathcal{D}_{Z}:=\right.$ $1 /(1+z) / \Gamma /\left(1-v_{\|} / c\right)$, where $\left.\Gamma:=(1-v / c)^{1 / 2}\right), 20$ logarithmically spaced nodes between 0.5 and 1.1 .

We determined these parameter ranges by analyzing the cosmological simulation data.

The first four parameters generate 681462 models that are computed by CLOUDY. For each model we assume a thermal equilibrium, use the CMB and HMO5 CLOUDY commands to generate the CMB and UV background radiation fields (Haardt and Madau 1996), and save the diffuse continuum. We then integrate the continuum in X-ray bands for each value of $\mathcal{D}_{Z}$. To be more specific, the soft X-ray emission is integrated between $0.5 / \mathcal{D}_{Z} \mathrm{keV}$ and $2 / \mathcal{D}_{Z} \mathrm{keV}$, while the hard band is between $2 / \mathcal{D}_{Z} \mathrm{keV}$ and $10 / \mathcal{D}_{Z} \mathrm{keV}$ (the division by $\mathcal{D}_{Z}$ simultaneously takes care of the redshift and the proper motion of emitting cells). This gives us $1.362924 \times 10^{7}$ final models organized in a 5D array. In general we use a five-way linear interpolation of the emissivity logarithm to predict the emission for arbitrary parameter combinations. However, in this particular case we do not interpolate in redshift, because the redshifts of our snapshots are the nodes of the table.

\section{A2 Interpolation accuracy}

To assess the accuracy of the interpolation table, we generated $10^{4}$ models using random combinations of parameters in the range mentioned in previous section. We then run CLOUDY to compute the spectrum of each model in the soft band, and then integrate it to obtain the correct emission in that band. Finally, we compare these values with those predicted by the table for the $10^{4}$ models.

The left panel in Fig. A1 shows a histogram of relative errors $\left|I_{\text {table }}-I_{\text {Cloudy }}\right| / I_{\text {Cloudy }}$, where $I_{\text {table }}$ and $I_{\text {Cloudy }}$ are the intensities in $0.5-2 \mathrm{keV}$ band predicted by the table and computed by Cloudy, respectively. The vertical lines denote the first quartile, median and the third quartile of the distribution. As can be seen, half of the errors are smaller than $0.35 \%$ and three-quarters of the errors are smaller than $8.6 \%$. We note that this is a very conservative estimate in that we used random values for each parameter independently. In our calculation the errors are expected to be much smaller. The right panel of Fig. A1 shows the distribution of errors when the interpolation parameters are varied. As can be seen, for temperatures above $10^{6} \mathrm{~K}$ the errors decrease significantly, while for the rest of the parameters there is no clear trend. Nevertheless, the accumulation around $0.35 \%$ is evident. We conclude that this interpolation table gives an acceptable tradeoff between the computational, memory and storage cost of running a large number of CLOUDY models and the interpolation accuracy.

Fig. A2 shows the dependence of table accuracy on the number of points. The gray line shows the results for a small interpolation table ( $\sim 250$ thousand points) that has very large errors. The medium-sized table ( $\sim 2$ million points) behaves better, but the median of its distribution is around $\sim 1 \%$. We decided that this is still a relatively large error, so we computed an even larger table ( $\sim 14$ million points) so that the median decreases to $\sim 0.35 \%$, which we deem acceptable for this application.

\section{A3 Comparison with thermal Bremsstrahlung}

We run a low-resolution image to compare the intensity distribution between the default SPEV thermal emission method (CuestaMartínez et al. 2015) and the new interpolation table based on CLOUdY.

Fig. A3 shows the unconvolved image pixel intensity distribution in soft X-rays. As can be seen, the distributions are quite similar for higher intensities (corresponding to higher temperatures), but the metal emission lines taken into account by CLOUDY produce consistently more intense emission at lower densities and temperatures than does the Cuesta-Martínez et al. 2015 model (which was not meant to be used in that regime). From this we conclude that the use of CLOUDY was warranted in this application, as it allowed us to reliably predict the emission from low-density and low-temperature media.

\section{APPENDIX B: COMPUTING THE SZ EFFECT. COMPARISON BETWEEN SPEV AND SZPACK}

In Section 3.3 we employed SPEV to compute the total, the thermal and the kinematic SZ signals associated to our selected cluster at different observational frequencies. However, when obtaining these synthetic observations, we avoided to account for relativistic corrections, which can be particularly relevant for high ICM temperatures $\left(T_{e}>10 \mathrm{keV}\right)$, large cluster peculiar velocities and high frequencies ( $x>10$; e.g. Nozawa et al. 2005). In order to get an impression of the relevance of this simplification, we compare here our results with those obtained with the publicly available library SZPACK (Chluba et al. 2012, 2013). Taking into account relativistic corrections, SZPACK allows for a precise estimation of the SZ effects in massive galaxy clusters for electron temperatures of up to $k T_{e} \sim 25 \mathrm{keV}$ and cluster peculiar velocities as large as $v / c \sim 0.01$.

To perform such a comparison we have considered the region of interest around our central galaxy cluster $(\sim 0.6$ squared degrees) and we have computed the total SZ effect with SPEV and with SZPACK at different frequencies. Now, to speed up the comparison, each map has been sampled with $600^{2}$ pixels, which provides a pixel size of $3.6 \times 3.6 \operatorname{arsec}^{2}$, a resolution $\sim 4$ times lower than the one employed in Section 3.3.

Figure B1 shows the absolute difference between the total SZ signal obtained with SPEV and with SZPACK at $\nu=128,217$ and $369 \mathrm{GHz}$ for the IGM (left panel) and the WHIM (right panel). In general, the distributions obtained for both gas phases are quite similar. As a function of the observation frequency, SPEV tends to overestimate positively the SZ effect at $369 \mathrm{GHz}$, whereas at lower frequencies the resulting signal tends to be negative. However, all distributions show a dominant peak around zero and are nearly symmetric. On average, $95 \%$ of the distributions have an absolute error for IGM (WHIM) below $0.16 \%$ (0.3\%), 4\% (2\%), and $325 \%(14 \%)$ at $\nu=128,217$ and $369 \mathrm{GHz}$, respectively. These results are quite encouraging, since they confirm that the synthetic total SZ maps generated with SPEV in the non-relativistic approximation are in a remarkably good agreement with the results obtained with SZPACK, at least at $\nu=128$ and $217 \mathrm{GHz}$. However, at the highest considered frequency the discrepancies generated between SPEV and SZPACK are more significant, especially for the IGM. According to Figs. 6 and 7, the total SZ signal obtained with SPEV at $\nu=369 \mathrm{GHz}$ is higher than at lower frequencies. In our selected volume, considering all the redshifts of interest, the average number of gas elements (cells) with a temperature above 10 

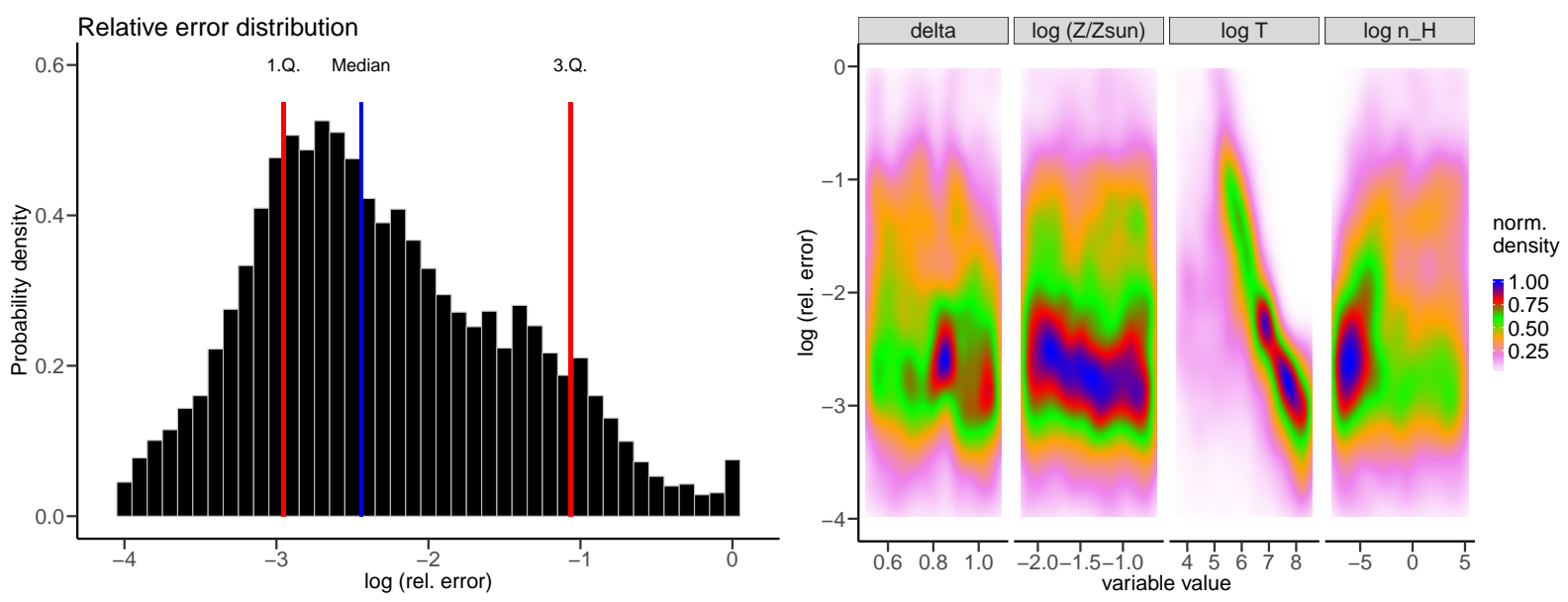

Figure A1. Relative errors of the soft X-ray emission predicted by the interpolation table and computed directly by CLOUDY for $10^{4}$ models whose parameters have been randomly drawn. Left: Relative error distribution histogram. The vertical lines denote the first quartile, the median and the third quartile of the distribution. Right: Dependence of relative errors on each of the four interpolation variables (note that in this test we fix the redshift $z$ ). The colors show the relative errors density distribution (separately normalized in each panel).

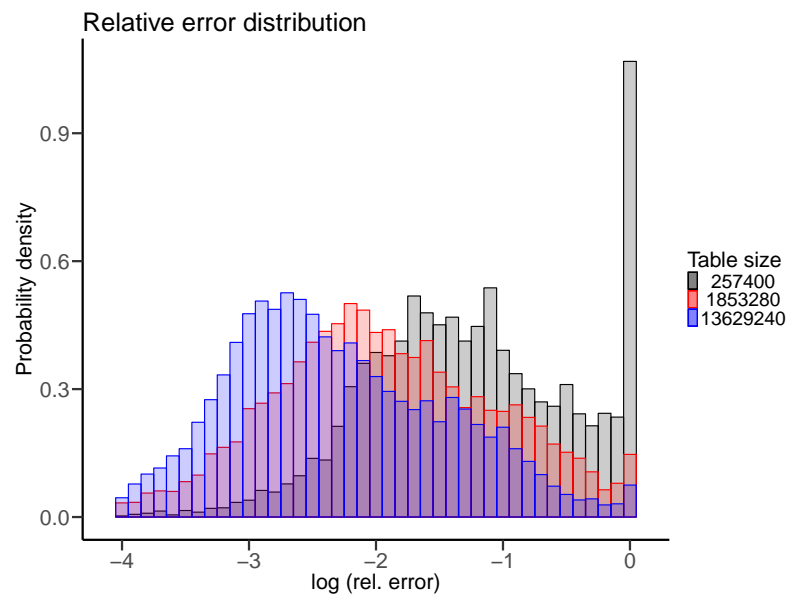

Figure A2. Relative errors of the soft X-ray emission predicted by different interpolation table sizes using $10^{4}$ test models. Gray, red and blue histograms show the error distribution for $2.574 \times 10^{5}, 1.85328 \times 10^{6}$ and $1.362924 \times 10^{7}$ points, respectively. All three tables cover the same parameter space.

$\mathrm{keV}$ is only $\sim 0.1 \%$. Moreover, our central cluster is moving with a velocity of $v / c \sim 0.003$. These arguments suggest that, in our case, increasing the frequency of observation could make it necessary to account for relativistic corrections. Indeed, at $\nu=369 \mathrm{GHz}$ $(x \sim 6.5)$ it seems already necessary to include these corrections in order to reproduce the results obtained with SZPACK.

Figure B2 shows the analysis of the differences between SPEV and SZPACK in four different SZ strength segments at 217 GHz. In each segment the difference has been normalized to the upper limit of that segment. While not being equivalent to relative differences (which could not be reliably computed since SPEV and SZPACK may give values of different signs or values close to zero when the SZ effect is small), this representation gives us a clue about the typical relative error we make by not taking into account the relativistic effects. The blue lines show the median in each segment, which is always below $10 \%$. The third quartiles are below $20 \%$. We note that the differences are smaller for pixels with stronger SZ effect.

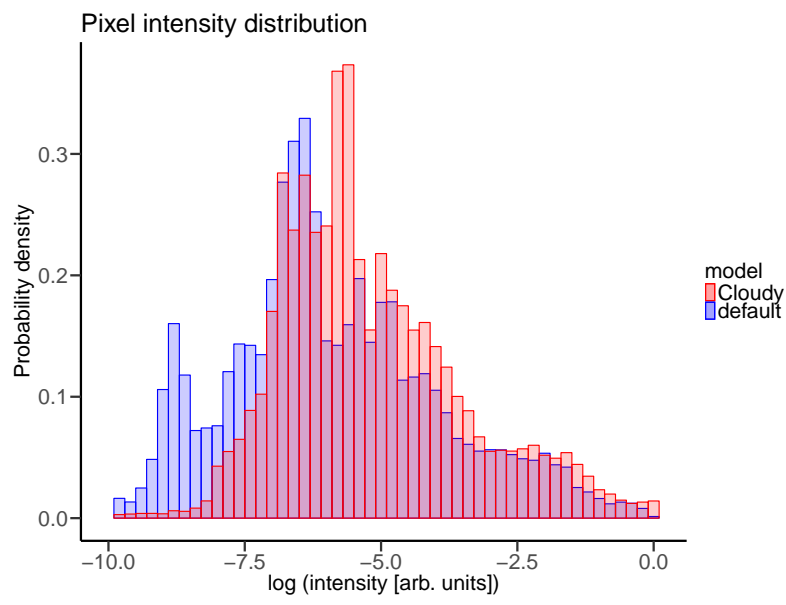

Figure A3. Distribution of unconvolved image pixel intensities (in arbitrary units) in the soft band $(0.5-2.0 \mathrm{keV})$ computed using the thermal emission as estimated by the method of Cuesta-Martínez et al. 2015, ("default", see Section 2.2.1 for details) and by an interpolation table based on CLOUDY ("Cloudy", see Section 2.2.1 for details).

With these tests in mind, we choose to keep using SPEV because most of the errors are not significant and because it allows us to optimize the calculation (SPEV runs in parallel). In a future work we plan to extend our analysis including the relativistic approach in SPEV and estimating the SZ effect for a larger sample of clusters. 

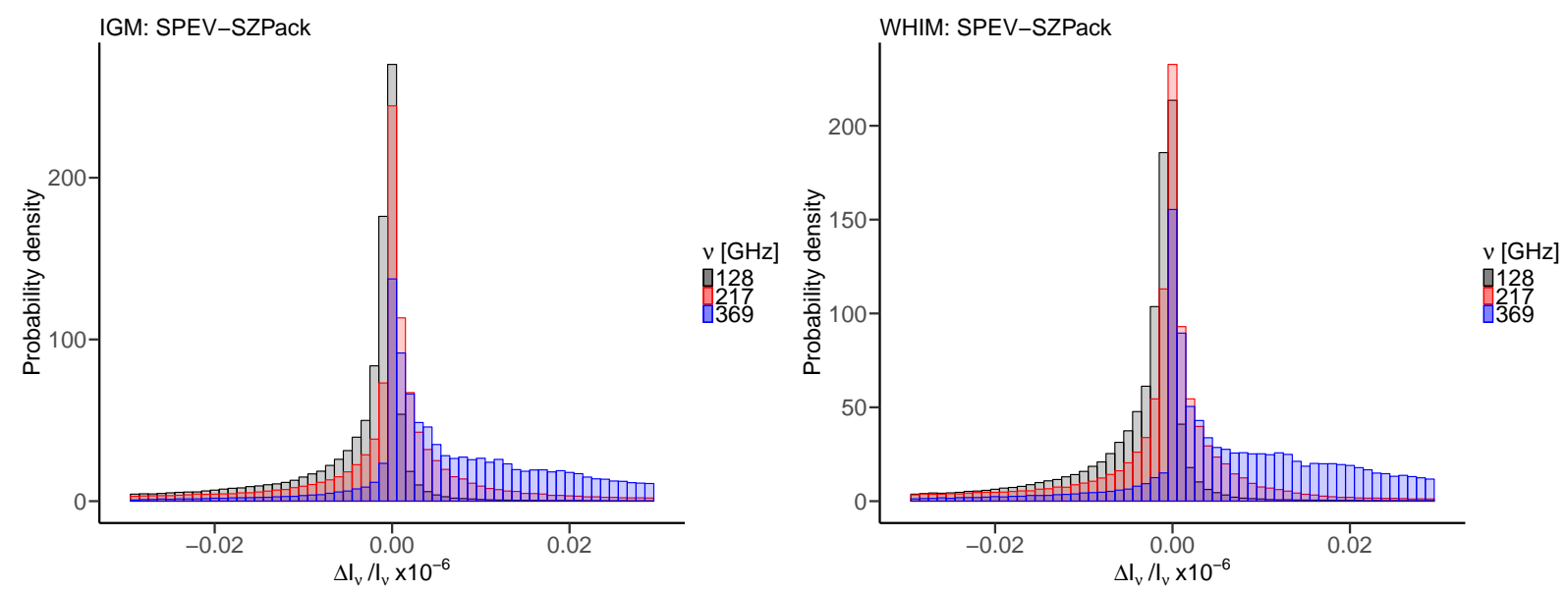

Figure B1. Absolute difference between the total SZ effect computed using SPEV and SZPACK at $\nu=128,217$ and 369 GHz for the IGM and the WHIM (left and right panels, respectively).

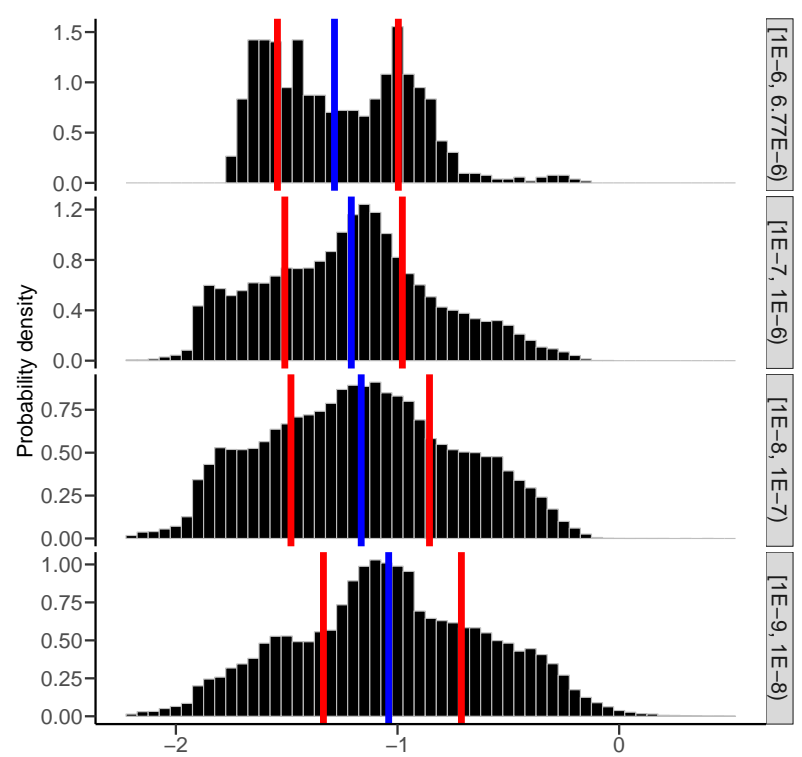

$\log (\mid$ difference| / seg. max.)

Figure B2. Normalized dfiferences between SPEV and SZPACK at 217 $\mathrm{GHz}$. The error distribution has been partitioned in four segments according to the size of the total SZ effect computed by SPEV. From top to bottom the segments are $\left[10^{-6}, 6.77 \times 10^{-6}\right),\left[10^{-7}, 10^{-6}\right),\left[10^{-8}, 10^{-7}\right)$ and $\left[10^{-9}, 10^{-8}\right)$. In each segment the absolute difference between SPEV and SZPACK has been divided by the upper limit of that segment. We show the distribution of the logarithm of that quantity. In addition, the blue line shows the median of each segment, while the red lines show the first and third quartiles. 